\title{
A Screen for Synaptic Growth Mutants Reveals Mechanisms That Stabilize Synaptic Strength
}

\author{
(DPragya Goel, ${ }^{1,2 *}$ Mehak Khan, ${ }^{1 *}$ (DSamantha Howard, ${ }^{1}$ Giwoo Kim, ${ }^{1}$ Beril Kiragasi, ${ }^{1,3}{ }^{\circ}$ Koto Kikuma, ${ }^{1,3}$ \\ and $\odot$ Dion Dickman ${ }^{1}$ \\ ${ }^{1}$ Department of Neurobiology, ${ }^{2}$ Graduate Program in Molecular and Computational Biology, and ${ }^{3}$ Neuroscience Graduate Program, University of Southern \\ California, Los Angeles, California 90089
}

\begin{abstract}
Synapses grow, prune, and remodel throughout development, experience, and disease. This structural plasticity can destabilize information transfer in the nervous system. However, neural activity remains stable throughout life, implying that adaptive countermeasures exist that maintain neurotransmission within proper physiological ranges. Aberrant synaptic structure and function have been associated with a variety of neural diseases, including Fragile X syndrome, autism, and intellectual disability. We have screened 300 mutants in Drosophila larvae of both sexes for defects in synaptic growth at the neuromuscular junction, identifying 12 mutants with severe reductions or enhancements in synaptic growth. Remarkably, electrophysiological recordings revealed that synaptic strength was unchanged in all but one of these mutants compared with WT. We used a combination of genetic, anatomical, and electrophysiological analyses to illuminate three mechanisms that stabilize synaptic strength despite major disparities in synaptic growth. These include compensatory changes in (1) postsynaptic neurotransmitter receptor abundance, (2) presynaptic morphology, and (3) active zone structure. Together, this characterization identifies new mutants with defects in synaptic growth and the adaptive strategies used by synapses to homeostatically stabilize neurotransmission in response.
\end{abstract}

Key words: homeostasis; neuromuscular junction; neurotransmission; plasticity; synapse; synaptic growth

\section{Significance Statement}

This study reveals compensatory mechanisms used by synapses to ensure stable functionality during severe alterations in synaptic growth using the neuromuscular junction of Drosophila melanogaster as a model system. Through a forward genetic screen, we identify mutants that exhibit dramatic undergrown or overgrown synapses yet express stable levels of synaptic strength, with three specific compensatory mechanisms discovered. Thus, this study reveals novel insights into the adaptive strategies that constrain neurotransmission within narrow physiological ranges while allowing considerable flexibility in overall synapse number. More broadly, these findings provide insights into how stable synaptic function may be maintained in the nervous system during periods of intensive synaptic growth, pruning, and remodeling.

\section{Introduction}

Dramatic changes in synapse number, morphology, and structure occur throughout nervous system development and during

\footnotetext{
Received Oct. 8, 2018; revised March 12, 2019; accepted March 14, 2019.

Author contributions: P.G. and D.D. designed research; P.G., M.K., S.H., G.K., B.K., and K.K. performed research; P.G., M.K., and S.H. analyzed data; P.G. and D.D. wrote the first draft of the paper; P.G., M.K., and D.D. edited the paper; P.G. and D.D. wrote the paper.

This work was supported by National Institutes of Health Grant NS091546 to D.D. M.K. and S.H. were supported by University of Southern California Undergraduate Research Awards. P.G. and K.K. were supported in part by University of Southern California Provost Graduate Research Fellowships. We thank Dr. Mitch McVey (Department of Biology, Tufts University) for sharing WRNexo mutants; the Developmental Studies Hybridoma Bank for antibodies used in this study; the Bloomington Drosophila Stock Center (National Institutes of Health P40D018537) for fly stocks; and Andrew Tung, Andrew An, Luke Nunnely, and Michelle Chee for contributions during early phases of this project.

The authors declare no competing financial interests.

*P.G. and M.K. contributed equally to this work.

Correspondence should be addressed to Dion Dickman at dickman@usc.edu.
}

various forms of plasticity and remodeling in the mature nervous system. Expansion and retraction of synaptic terminals contribute to the refinement of neural circuits during developmental pruning, sleep/wake behavior, and experience-dependent plasticity (Cohen-Cory, 2002; Holtmaat and Svoboda, 2009; Riccomagno and Kolodkin, 2015; de Vivo et al., 2017). While these dynamic changes enable the flexibility necessary to wire the nervous system during development and to modify synapses during learning and memory, they pose a major challenge to the stability of neural function. Indeed, it is interesting to note that the period of highest susceptibility to seizures occurs during the first years of life, a period of dramatic synaptic growth and pliability in the nervous system (Holmes and Ben-Ari, 1998; Baram, 2012). 
Despite the potential for these processes to disrupt coherent information transfer, homeostatic mechanisms maintain physiologically stable levels of functionality (Pozo and Goda, 2010; Turrigiano, 2017).

The Drosophila neuromuscular junction (NMJ) is a powerful model system to illuminate the genes and mechanisms that integrate synaptic growth, function, and homeostatic plasticity. At this model glutamatergic synapse, stereotyped levels of synaptic strength are consistently observed despite a dramatic expansion in synaptic growth, where the NMJ rapidly enlarges by $\sim 100$-fold during larval development (Atwood et al., 1993; Schuster et al., 1996). Remarkably, neurotransmission is maintained within narrow physiological ranges during this process (Davis and Goodman, 1998b), implying that adaptive forms of plasticity stabilize synaptic function in coordination with growth. This robust scaling of synaptic growth with muscle target growth has also been shown to homeostatically maintain muscle excitation in classical studies in crayfish (Lnenicka and Mellon, 1983a,b). A variety of homeostatic mechanisms are triggered at the Drosophila NMJ in response to excess glutamate release (Daniels et al., 2004; Gavino et al., 2015; Li et al., 2018a), diminished postsynaptic neurotransmitter receptor functionality (Petersen et al., 1997; Frank et al., 2006), injury-related signaling (Goel and Dickman, 2018), and biased innervation (Davis and Goodman, 1998a; Goel and Dickman, 2018). These mechanisms can operate with specificity at individual motor inputs and synapses (Davis and Goodman, 1998a; Newman et al., 2017; Li et al., 2018b). There is also evidence that additional homeostatic processes stabilize global synaptic strength when total synapse numbers are drastically altered at the NMJ. For example, it has been estimated that as many as $44 \%$ of the genes encoded in the Drosophila genome influence synaptic growth and structure (Liebl et al., 2006), while far fewer genes appear to be involved in neurotransmission (Dickman and Davis, 2009; Müller et al., 2011). The mechanisms that serve to stabilize global synaptic strength when confronted with variations in synaptic growth have yet to be defined.

Mutations in genes linked to neurological and neuropsychiatric diseases are attractive candidates to screen for defects in synaptic growth, structure, and plasticity. Indeed, aberrations in these processes are associated with a variety of neural diseases, including Fragile X syndrome, autism spectrum disorder, schizophrenia, and intellectual disability (Zoghbi and Bear, 2012; Wondolowski and Dickman, 2013; Forrest et al., 2018). We have therefore screened a collection of mutations in genes with links to these neural diseases for roles in synaptic growth and transmission at the Drosophila NMJ, discovering new mutants with defects in these processes. Interestingly, this approach also confirmed that, while synaptic growth can vary considerably across mutations in diverse genes, neurotransmission is constrained within much narrower physiological ranges. Given these results, we chose not to characterize in detail the specific functions of individual genes in regulating synaptic growth. Rather, we investigated synaptic structure and function in the subset of mutants that exhibited the most extreme changes in synaptic growth but that, remarkably, maintained stable synaptic strength. This effort defined three mechanisms that targeted both presynaptic and postsynaptic structures for homeostatic modulation. Together, these results elucidate adaptive strategies that can be used by synapses to maintain set point levels of synaptic strength when confronted with extreme alterations to synaptic growth.

\section{Materials and Methods}

Fly stocks. Drosophila stocks were raised at $25^{\circ} \mathrm{C}$ on standard molasses food. The $w^{1118}$ strain (RRID:BDSC_5905) is used as the WT control unless otherwise noted, as this is the genetic background of the genetic mutants used in this study. For experiments with the transgenic RNAi lines, control larvae were generated by crossing C15 (c155-Gal4;ScaGal4;BG57-Gal4) (Brusich et al., 2015) to UAS-RFP (BDSC \#32218; RRID:BDSC_32218). Because the average synaptic growth and electrophysiological values for the mutant control $\left(w^{1118}\right)$ and RNAi control (c155-Gal4;Sca-Gal4/+;BG57-Gal4/UAS-RFP) were not significantly different (Table 1-1, available at https://doi.org/10.1523/JNEUROSCI. 2601-18.2019.t1-1), we pooled all mutant and RNAi line data shown in Figures 1, 2, and 3. The WRNexo null mutants (WRNexo ${ }^{\mathbf{\Delta}}$ ) were previously described (Bolterstein et al., 2014). All other genetic mutants and transgenic RNAi lines were obtained from the Bloomington Drosophila Stock Center (BDSC). All experiments were performed on male or female third-instar larvae. A complete list of all stocks used in this study, their full genotypes, and their origin can be found in Table 1-1 (available at https://doi.org/10.1523/JNEUROSCI.2601-18.2019.t1-1).

Immunocytochemistry. Third-instar male or female larvae were dissected in ice-cold $0 \mathrm{Ca}^{2+} \mathrm{HL}-3$ and fixed in Bouin's fixative for $5 \mathrm{~min}$ as described previously (Chen et al., 2017). Larvae were washed with PBS containing $0.1 \%$ Triton X-100 (PBST) for $30 \mathrm{~min}$, and then blocked for $1 \mathrm{~h}$ with $5 \%$ normal donkey serum in PBST. Larvae were incubated overnight in primary antibodies at $4^{\circ} \mathrm{C}$ followed by a $30 \mathrm{~min}$ wash in PBST, $2.5 \mathrm{~h}$ incubation in secondary antibodies at room temperature $\left(20^{\circ} \mathrm{C}-22^{\circ} \mathrm{C}\right)$, a final $30 \mathrm{~min}$ wash in PBST, and equilibration in $70 \%$ glycerol. Blocking was done with 5\% normal donkey serum in PBST. Samples were mounted in VectaShield (Vector Laboratories). The following antibodies were used: mouse anti-Bruchpilot (nc82; 1:100; Developmental Studies Hybridoma Bank; RRID:AB_2314866); rabbit antiDLG (1:10,000) (Pielage et al., 2005); guinea pig anti-vGlut (1:2000) (Goel and Dickman, 2018); mouse anti-GluRIIA (8B4D2; 1:100; Developmental Studies Hybridoma Bank; RRID:AB_528269); rabbit antiGluRIIB (1:1000), generated by Cocalico Biologicals using the peptide described by Qin et al. (2005); and guinea pig anti-GluRIID (1:1000) (Perry et al., 2017). Donkey anti-mouse, anti-guinea pig, and anti-rabbit AlexaFluor-488-, Cyanine 3 (Cy3)-, and Dy Light 405-conjugated secondary antibodies (Jackson ImmunoResearch Laboratories) were used at 1:400. AlexaFluor-647 conjugated goat anti-HRP (Jackson ImmunoResearch Laboratories) was used at 1:200.

Imaging and analysis. Samples were imaged using a Nikon A1R Resonant Scanning Confocal microscope equipped with NIS Elements software and a $100 \times$ APO 1.4 NA oil-immersion objective using separate channels with four laser lines $(405,488,561$, and $637 \mathrm{~nm})$. For fluorescence quantifications of Bruchpilot (BRP) intensity levels, $z$ stacks were obtained using identical settings for all genotypes with $z$ axis spacing between 0.15 and $0.2 \mu \mathrm{m}$ within an experiment and optimized for detection without saturation of the signal as described. Boutons were counted using vGlut and HRP-stained NMJ terminals on muscle 6/7 and muscle 4 of segment A3, considering each vGlut puncta to be a bouton. Consistent changes in bouton numbers were observed across both muscle types (Table 4-1, available at https://doi.org/10.1523/JNEUROSCI.2601-18. 2019.t4-1). The general analysis toolkit in the NIS Elements software was used for image analysis as described previously (Kikuma et al., 2017). Neuronal surface area was calculated by creating a mask around the HRP channel that labels the neuronal membrane. BRP puncta number, area, and total BRP intensity per NMJ were quantified by applying intensity thresholds and filters to binary layers on the BRP labeled 488 channel as described previously (Goel et al., 2019b). GluRIIA, GluRIIB, and GluRIID puncta intensities were quantified by measuring the total sum intensity of each individual GluR puncta, and these values were then averaged per NMJ to get one reading $(n)$. Measurements based on confocal images were taken from at least 12 synapses acquired from at least 6 different animals.

Electrophysiology. All dissections and recordings were performed in modified HL-3 saline (Stewart et al., 1994; Dickman et al., 2005; Kiragasi et al., 2017) containing the following (in $\mathrm{mm}$ ): $70 \mathrm{NaCl}, 5 \mathrm{KCl}, 10 \mathrm{MgCl}_{2}$, 
$10 \mathrm{NaHCO}_{3}, 115$ sucrose, 5 trehelose, 5 HEPES, and $0.4 \mathrm{CaCl}_{2}, \mathrm{pH}$ 7.2. Neuromuscular junction sharp electrode (electrode resistance between 10 and $30 \mathrm{M} \Omega$ ) recordings were performed on muscles 6 and 7 of abdominal segments A2 and A3 in wandering third-instar larvae as described previously (Goel et al., 2019a). Consistent changes in values of mEPSP, EPSP, and quantal content were observed at muscle 4 across genotypes (Table 4-1, available at https://doi.org/10.1523/JNEUROSCI.2601-18. 2019.t4-1). Briefly, larvae were dissected and loosely pinned; the guts, trachea, and ventral nerve cord were removed from the larval body walls with the motor nerve cut, and the preparation was perfused several times with HL-3 saline. Recordings were performed on an Olympus BX61 WI microscope using a $40 \times / 0.80$ water-dipping objective, and acquired using an Axoclamp 900A amplifier, Digidata 1440A acquisition system, and pClamp 10.5 software (Molecular Devices). Electrophysiological sweeps were digitized at $10 \mathrm{kHz}$ and filtered at $1 \mathrm{kHz}$. Data were analyzed using Clampfit (Molecular Devices), MiniAnalysis (Synaptosoft), and Excel (Microsoft) software.

mEPSPs were recorded in the absence of any stimulation, and cut motor axons were stimulated to elicit EPSPs. An ISO-Flex stimulus isolator (A.M.P.I.) was used to modulate the amplitude of stimulatory currents. Intensity was adjusted for each cell, set to consistently elicit responses from both neurons innervating the muscle segment, but avoiding overstimulation. Average mEPSP, EPSP, and quantal content were calculated for each genotype by dividing EPSP amplitude by mEPSP amplitude. Muscle input resistance $\left(\mathrm{R}_{\mathrm{in}}\right)$ and resting membrane potential $\left(V_{\text {rest }}\right)$ were monitored during each experiment. Recordings were rejected if the $V_{\text {rest }}$ was $>-60 \mathrm{mV}$, if the $R_{\text {in }}$ was $<5 \mathrm{~m} \Omega$, or if either measurement deviated by $>10 \%$ during the course of the experiment. Larvae were incubated with or without philanthotoxin-433 (PhTx; Sigma-Aldrich; $20 \mu \mathrm{M}$ ) resuspended in HL-3 for $10 \mathrm{~min}$, as described previously (Frank et al., 2006; Dickman and Davis, 2009).

Experimental design and statistical analysis. For electrophysiological and immunostaining experiments, each NMJ terminal (muscle 6 for physiology, and muscle 4 for immunostaining analyses of synaptic terminals and active zones) is considered an $n$ of 1 because each presynaptic motor neuron terminal is confined to its own muscular hemi-segment. For these experiments, muscles 4 or 6 were analyzed from hemi-segments A3 for each larvae, and thus each larvae contributes 2 NMJs per experiment. To control for variability between larvae within a genotype, for immunostaining experiments involving BRP and glutamate receptors, NMJs were analyzed from at least 6 individual larvae. In the mutants characterized in Figures 4, 5, 6, 7, and 8, we observed similar differences in synaptic structure or function between NMJs on muscles 6 and 4 (Table 4-1, available at https://doi.org/10.1523/JNEUROSCI.260118.2019.t4-1).

Statistical analysis was performed using GraphPad Prism (version 7.0) or Microsoft Excel software (version 16.22). Data were tested for normality using a D'Agostino-Pearson omnibus normality test, which determined that the assumption of normality of the sample distribution was not violated. Normally distributed data were analyzed for statistical significance using a $t$ test (pairwise comparison), or an ANOVA and Tukey's test for multiple comparisons. Data were then compared using either a one-way ANOVA and tested for significance using a Tukey's multiple comparison test or using an unpaired two-tailed Student's $t$ test with Welch's correction. All data are presented as mean \pm SEM with varying levels of significance. For additional statistical details and values, see Table 4-1 (available at https://doi.org/10.1523/JNEUROSCI.2601-18. 2019.t4-1).

\section{Results}

\section{A forward screen identifies mutants with aberrant synaptic growth and transmission at the Drosophila NMJ}

To systematically screen a collection of mutations for defects in synaptic growth and function, we first established a list of Drosophila homologs of mammalian genes linked to synaptic function and neural disease. The initial list consisted of $\sim 800$ mammalian genes encoding proteins present at synapses and/or linked with neural disease (Table 1-1, available at https://doi.org/
10.1523/JNEUROSCI.2601-18.2019.t1-1). A subset of these genes encoded putative transcripts associated with FMRP (Darnell et al., 2011; Thomson et al., 2017), whereas additional genes were selected because of associations with schizophrenia and autism spectrum disorder (Gilman et al., 2011; Sando et al., 2012; Jurado et al., 2013; Serretti and Fabbri, 2013). From this list, we identified a final group of 300 Drosophila homologs: 132 putative FMRP targets and 168 genes encoding proteins present at synapses and/or associated with other diseases. From this initial list, we obtained a collection of 109 putative genetic mutations and 191 RNAi lines from public resources (Table 1-1, available at https://doi.org/10.1523/JNEUROSCI.2601-18.2019.t1-1). Finally, we assessed the lethal phase of homozygous mutants and RNAi lines crossed to NMJ drivers, removing any that failed to survive to at least the third-instar larval stage. Together, this effort established a collection of 297 mutants to screen for defects in synaptic growth and function at the third-instar larval NMJ.

We first assessed synaptic growth in this collection of 297 mutants and RNAi lines. Specifically, we characterized homozygous mutants or larvae in which RNAi transgenes were driven in both motor neurons and muscle (see Materials and Methods) (Brusich et al., 2015). Immunostaining of synaptic boutons at the Drosophila NMJ was used to quantify synaptic growth. WT NMJs typically exhibit $\sim 30$ boutons at the muscle 4 synapse (Fig. $1 A, B, D)$. We immunostained the NMJ with markers for synaptic vesicles (vGlut) and the neuronal membrane (HRP), and considered a single puncta of vGlut intensity to represent a synaptic bouton (Fig. 1 $A, B$ ). Quantification of bouton numbers across all 297 mutants and RNAi lines revealed a broad distribution, with 31.2 boutons as the mean and a SD of 6.8 (Fig. 1D). From this analysis, we selected the subset of mutants or RNAi lines that displayed the most extreme difference in bouton number, using 2 SDs above or below the mean $(>44 \%$ increase or decrease; Fig. $1 C, D)$ as cutoffs for further study.

Twelve mutants with extreme changes in synaptic growth at the NMJ were identified (Fig. 1C,D). All 12 were genetic mutants; four exhibited a reduction of $>44 \%$ in bouton number and were termed "undergrowth mutants" (Fig. 1C-E, blue), whereas the other eight exhibited an increase of $>44 \%$ in bouton number and were termed "overgrowth mutants" (Fig. $1 C-E$, red). Of the 12 positive hits from our initial screen, three genes were previously reported to have defects in synaptic growth (Fig. $1 D, E$ ), serving to validate our approach. These include the G-protein-coupled receptor flamingo (Bao et al., 2007), the serine-threonine kinase Akt1 (Natarajan et al., 2013), and the translation factor eIF-4E (Sigrist et al., 2003). Thus, from this initial screen of 297 lines, we identified four undergrowth and five overgrowth genes that have not previously been reported to regulate synaptic growth. The putative functions of these genes are detailed in Table 1-2 (available at https://doi.org/10.1523/JNEUROSCI.2601-18.2019.t1-2).

We also assayed synaptic transmission in the collection of 297 lines (Fig. 2A). We used electrophysiology to quantify mEPSP amplitude and evoked EPSP amplitude, and to calculate the number of synaptic vesicles released per stimulus (quantal content, a measure of neurotransmitter release) from each mutant screened (Table 1-1, available at https://doi.org/10.1523/ JNEUROSCI.2601-18.2019.t1-1). Electrophysiological recordings from all mutant and RNAi lines revealed a mean EPSP amplitude of $35.4 \mathrm{mV}$ and an SD of $6.5 \mathrm{mV}$ (Fig. 2B). We identified 40 mutant and RNAi lines with EPSP amplitudes $>2$ SDs below the mean ( $>36 \%$; Fig. $2 A, B$ ), although no targets exhibited an increase in EPSP amplitude of $>36 \%$ relative to the mean (Fig. 2A; Table 1-1, available at https://doi.org/10.1523/ 
A

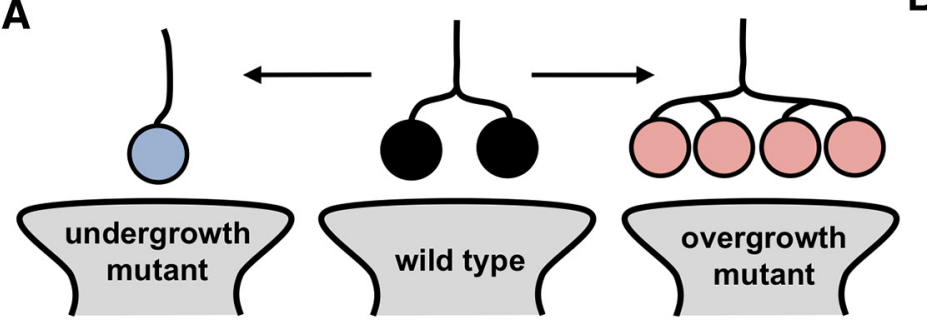

B

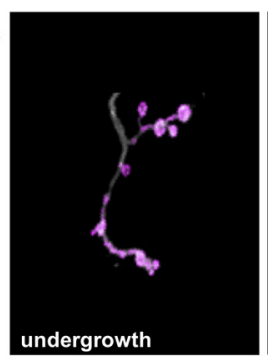

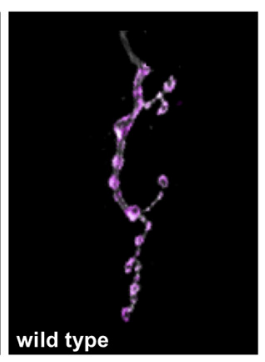

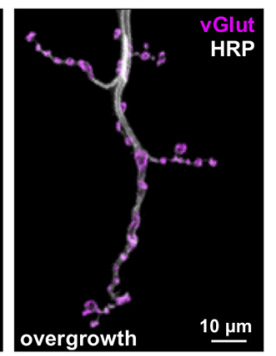

C

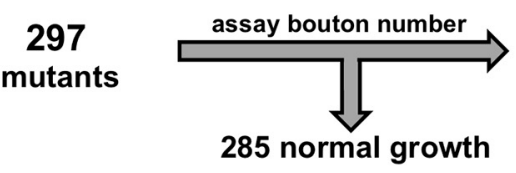

D

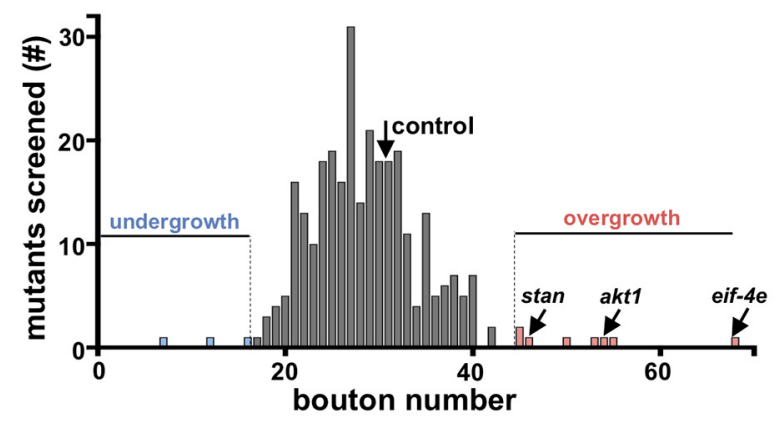

12 altered growth

\section{E}

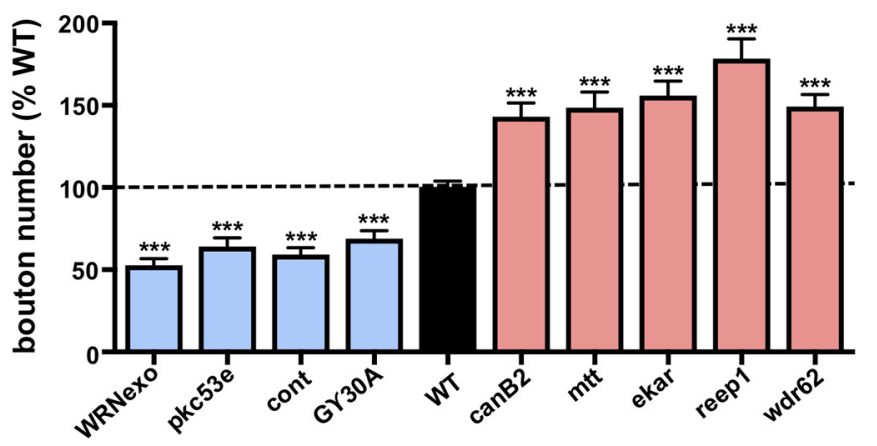

Figure 1. A forward genetic screen identifies synaptic growth mutants at the Drosophila NMJ. $A$, Schematic illustrating synaptic boutons, numbers of which are a measure of NMJ growth. $B$, Images of larval muscle 4 NMJs immunostained with anti-HRP (neuronal membrane marker) and anti-vGlut (synaptic vesicle marker). Examples of NMJs in undergrowth and overgrowth mutants are shown. C, Flow diagram of synaptic growth screen strategy and outcome. Mutants with increases or decreases in synaptic growth that were $>2$ SDs from controls ( $\sim 44 \%$ increase or decrease) are indicated. $\boldsymbol{D}$, Histogram of average bouton number per mutant or RNAi line quantified in the synaptic growth screen. Average bouton numbers in control (black arrow), overgrowth mutants (red), and undergrowth mutants (blue) are indicated. Three genes previously reported to exhibit synaptic overgrowth are indicated. $\boldsymbol{E}$, Bouton numbers of the identified overgrowth and undergrowth mutants shown as a percentage of WT values. No significant differences in bouton numbers were observed between the mutant control $\left(w^{1118}\right)$ and RNAi line control $(C 15 \times U A S-R F P ;$ Table 1-1, available at https://doi.org/10.1523/JNEUROSCI.2601-18.2019.t1-1) and were pooled. Error bars indicate \pm SEM. ${ }^{* * *} p \leq 0.001$. Additional details of all mutants and RNAi lines screened and statistical information (mean values, SEM, $n, p$ ) are shown in Table 1-1 (available at https://doi.org/10.1523/JNEUROSCI.2601-18.2019.t1-1) and Table 1-2 (available at https://doi.org/10.1523/JNEUROSCI.2601-18.2019.t1-2).

JNEUROSCI.2601-18.2019.t1-1). Quantification of bouton numbers in the 40 synaptic transmission mutants or RNAi lines revealed values similar to WT (Fig. 2C), consistent with previous studies that have shown that aberrant synaptic function often occurs without any major defects in synaptic growth (Kittel et al., 2006; Dickman and Davis, 2009; Reddy-Alla et al., 2017). This suggests that defects in synaptic function alone, independently of reduced growth, disrupts neurotransmission in these lines.

\section{Synaptic strength remains constant despite variations in} synaptic growth

We next focused on understanding how synaptic function remains stable across the broad variation in synaptic growth by analyzing synaptic growth and structure in the 257 remaining mutants and RNAi lines with relatively stable EPSP amplitudes. First, we considered two possible models to describe the relationship between synaptic growth (bouton numbers) and synaptic strength (EPSP amplitude). In a "scaling" model, each individual bouton operates as an independent unit of synaptic function, with all boutons functionally equivalent (Fig. $3 A$ ). Hence, synaptic strength would be predicted to scale in amplitude in proportion to the total number of synaptic boutons, with the number of individual synapses (active zone and glutamate receptor dyads) linearly increasing with the number of boutons. Assuming the functionality of each dyad to be constant, as bouton number increases or decreases, total synaptic strength would scale accordingly (Fig. 3A). Alternatively, in a "homeostatic" model, synapses would be adaptively modulated to counteract variations in synaptic growth and maintain stable levels of global synaptic strength (Fig. 3B). In this case, adaptations in total active zone number, presynaptic release probability, and/or postsynaptic receptivity to neurotransmitter would compensate for altered bouton number to tune synaptic strength and maintain constant levels of neurotransmission. We tested whether a scaling or homeostatic model best described our data from the genetic screen.

We plotted the average EPSP amplitude of each mutant screened as a function of total bouton number for that specific mutant (Fig. 3C). A scaling model would predict a linear relationship in this plot, where synaptic strength (EPSP amplitude) is proportional to bouton number (Fig. 3C, dotted line). However, this analysis found no significant correlation between EPSP amplitude and bouton number $\left(R^{2}=0.0002, p=0.7935\right)$. Rather, the majority of mutants screened (86\%) maintained EPSP amplitudes between a narrow range of $32-36 \mathrm{mV}$ (Fig. 3C), more consistent with a homeostatic model. Next, we examined synaptic strength in the most extreme four undergrowth and five over- 
A
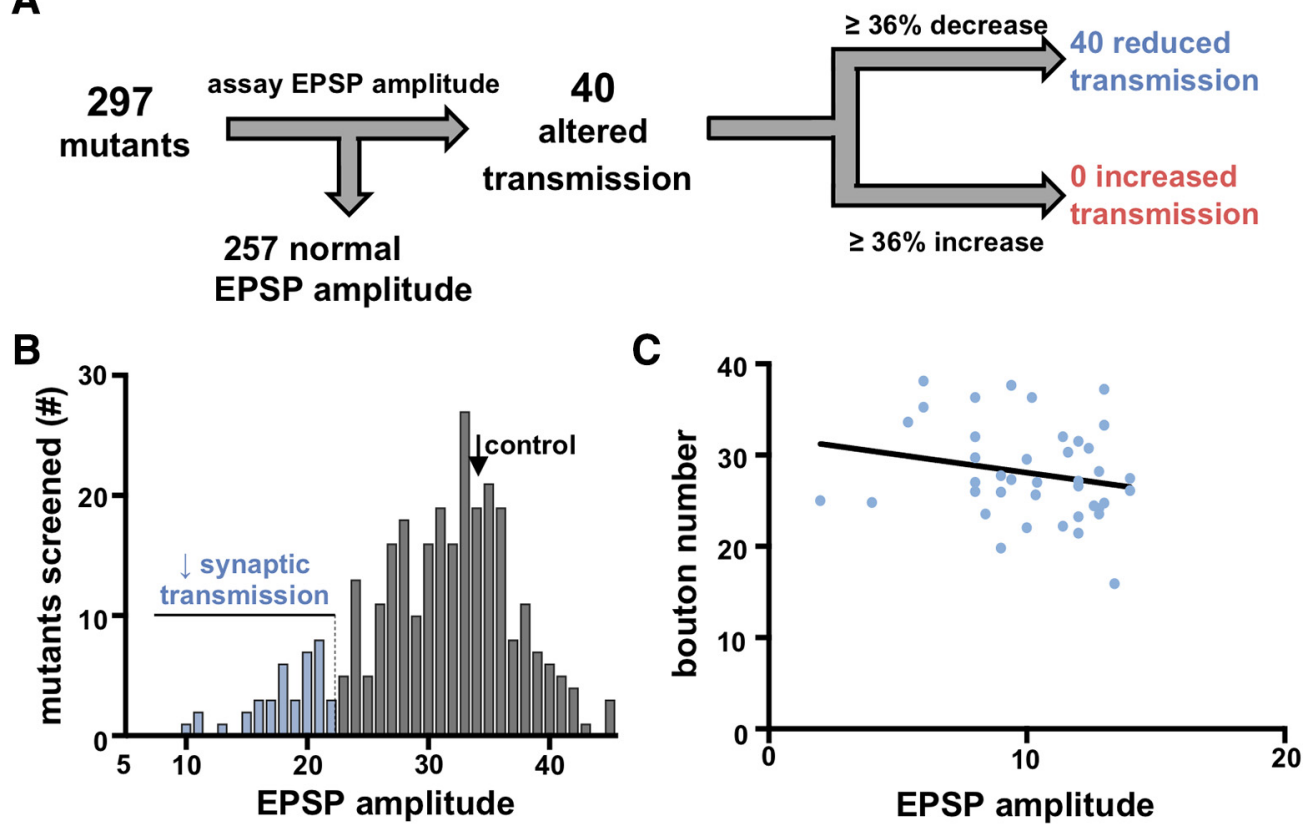

Figure 2. Presynaptic neurotransmitter release does not scale with synaptic growth in the mutants screened. $\boldsymbol{A}$, Flow diagram of electrophysiology-based synaptic transmission screen strategy and outcome. $\boldsymbol{B}$, Histogram of average EPSP amplitude quantified for each mutant and RNAi line in the screen. Although no mutants or RNAi lines with EPSP amplitudes $>2$ SDs above the average EPSP amplitude in WT were found ( $\sim 36 \%$ increase), several lines with EPSP amplitudes below this threshold were identified (represented as blue). $C$, Graph showing the total bouton number of each synaptic transmission mutant or RNAi line identified as a function of EPSP amplitude. The best-fit line to these data (solid black line; slope $=-0.788$ ) indicates that bouton numbers do not correlate with EPSP amplitude $\left(R^{2}=0.045, p=0.186\right)$. Additional details of all mutant and RNAi lines screened and statistical information (mean values, SEM, $\left.n, p\right)$ are shown in Table $1-1$ (available at https://doi.org/10.1523/JNEUROSCl.2601-18.2019.t1-1).

growth mutants discussed in Figure 1E. We plotted the EPSP amplitude for each mutant as a function of bouton number (Fig. $3 D$ ). Interestingly, all but one of the nine mutants exhibited EPSP amplitudes consistent with a homeostatic model, whereas one mutant, pkc53E, best fit with a scaling model. Finally, we considered that for a homeostatic model to be truly "homeostatic," presynaptic neurotransmitter release (quantal content) for each individual bouton should inversely scale with total boutons per NMJ. Indeed, when the average quantal content was normalized per bouton for all 257 mutants and RNAi lines, a robust scaling of quanta released per bouton was observed (Fig. $3 E$ ), consistent with a homeostatic tuning of presynaptic release per bouton. Together, this analysis of synaptic growth and function in the mutants screened is consistent with the homeostatic model schematized in Figure 3B, suggesting that presynaptic neurotransmitter release is tuned at individual boutons to maintain stable global synaptic strength despite variation in synaptic growth.

\section{Synaptic strength scales with synaptic growth in pkc53E mutants}

We next characterized the relationship between synaptic growth and function in the nine synaptic growth mutants in more detail, seeking to illuminate what processes stabilized synaptic strength in eight of nine mutants. We first assessed synaptic function and structure in the four undergrowth mutants. Mutations in the first gene, protein kinase C 53E ( $p k c 53 E$ ), exhibited reductions in synaptic strength that appeared to scale with synaptic growth (Fig. $3 D)$. Bouton numbers were reduced by $\sim 50 \%$ in homozygous mutants of $p k c 53 E$ and in $p k c 53 E$ mutants in trans to a deficiency that removed the entire locus ( $p k c 53 E^{1} / p k c 53 E^{D f}$; Fig. $4 A, B$ ). Correspondingly, EPSP amplitude was reduced to a similar extent in both allelic combinations of pkc53E compared with WT (Fig. 4C,D). Synaptic strength, indicated by EPSP amplitude, is determined by two parameters: the amount of presynaptic neurotransmitter released and the postsynaptic response (Del Castillo and Katz, 1954; Lisman et al., 2007). A change in mEPSP amplitude, which reflects the postsynaptic response to neurotransmitter released from a single vesicle, would likely indicate a change in the number or functionality of postsynaptic glutamate receptors in $p k c 53 E$ mutants. However, we observed no significant difference in mEPSP amplitude in $p k c 53 E$ mutants compared with WT (Fig. 4D), consistent with no postsynaptic adaptations in this mutant. Next, we calculated quantal content in these mutants, a measure of the number of synaptic vesicles released in response to synaptic stimulation. We found a reduction in this value proportional to the reduction in EPSP amplitude (Fig. 4C,D), as expected. If no adaptions to presynaptic structure occurred in $p k c 53 E$, then the anatomical number of release sites (active zones) should be reduced in proportion to the reduction in bouton number. We measured the number of puncta of the active zone scaffold BRP by immunostaining the NMJ, which represent individual releases sites (Kittel et al., 2006; Wagh et al., 2006). We observed a reduction in BRP puncta number per NMJ proportional to the reduction in bouton number in pkc53E mutants (Fig. 4E, F), with no change in BRP puncta density compared with WT (Fig. $4 F$ ). Thus, in $p k c 53 E$ mutants, the number of active zones is reduced in proportion to the number of boutons and no apparent changes are observed in release probability or the postsynaptic sensitivity to neurotransmitter, consistent with a scaling of synaptic strength with synaptic growth. Importantly, this implies that, in the remaining eight mutants in which synaptic strength remained constant despite increased or reduced growth, some compensatory adaptions must have occurred.

The observation that $p k c 53 E$ mutants failed to express any apparent forms of adaptive plasticity to sustain normal muscle 
A Neurotransmitter release scales with bouton number

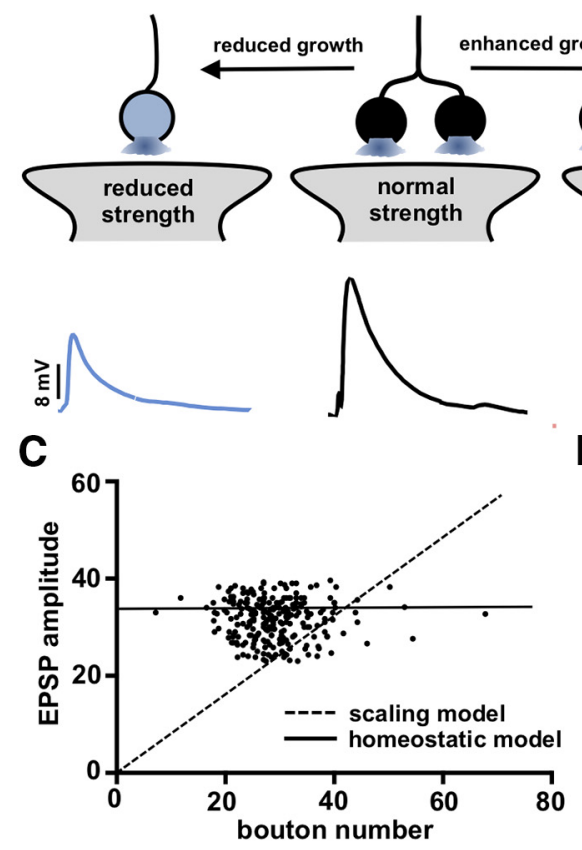

D
B Homeostatic adaptations stabilize neurotransmission
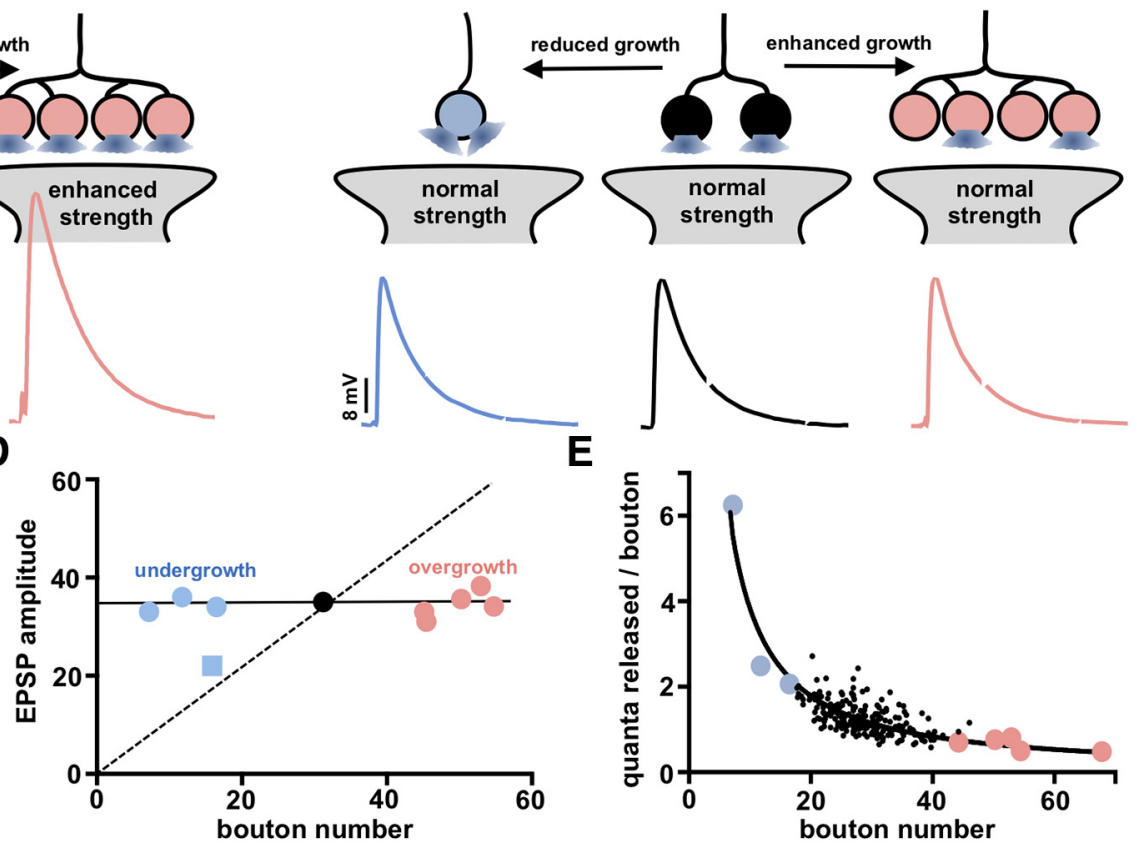

E

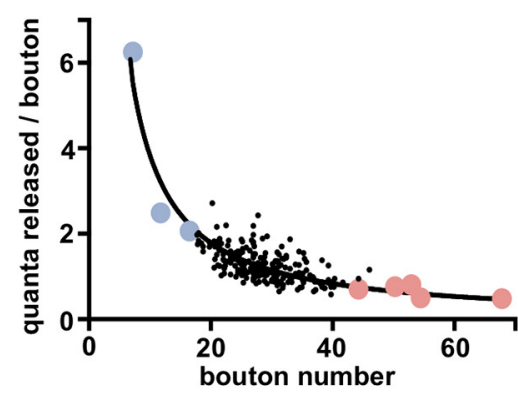

Figure 3. Stable synaptic strength is observed in the mutants screened despite variation in synaptic growth. $\boldsymbol{A}$, Schematic illustrating a "scaling" model in which presynaptic neurotransmitter release scales with synaptic growth. In this scenario, EPSP amplitude correlates with bouton number. $\boldsymbol{B}$, Schematic illustrating an alternative "homeostatic" model, in which synaptic strength remains constant across changes in bouton number. C, Graph plotting the EPSP amplitude of the genes screened (with the mutants and RNAi lines defective in synaptic transmission removed) plotted as a function of bouton number. Dashed diagonal line indicates the ideal "scaling" model, where EPSP amplitude correlates with bouton numbers. Horizontal solid line indicates the idealized "homeostatic" model, where no such correlation is observed. The data show that EPSP amplitudes do not correlate with bouton numbers (Pearson's correlation coefficient $R^{2}=0.0002, p=0.789$ ), a closer fit to a "homeostatic" model. D, Graph plotting EPSP amplitude of the synaptic overgrowth and undergrowth mutants as a function of bouton number. Only a single undergrowth mutant (indicated as a square data point) fits the "scaling" model, with EPSP amplitude reduced to a similar extent as the reduction in bouton number. All other synaptic growth mutants maintained stable EPSP amplitude, consistent with a "homeostatic" model (solid horizontal line; Pearson's correlation coefficient $R^{2}=0.012, p=0.718$ ). $E$, Average quanta released per bouton calculated for each mutant are plotted as a function of bouton number for the mutants shown in $C$. A curve fit of these data provides a goodness-0f-fit $R^{2}$ value of 0.65 and a $p$ value of $<0.0001$, indicating an inverse correlation between quanta released per bouton with total bouton number. Additional details of the mutants screened and statistical information (mean values, SEM, $n, p$ ) are shown in Table $1-1$ (available at https://doi.org/10.1523/JNEUROSCI.2601-18.2019.t1-1).

excitation led to us assess whether these mutants were capable of expressing homeostatic plasticity. Although multiple and diverse forms of homeostatic plasticity can be simultaneously expressed and balanced at the Drosophila NMJ (Gavino et al., 2015; Li et al., 2018a; Goel et al., 2019b), there is evidence that in some cases a hierarchy between different modes of plasticity exist where expression of one form occludes expression of another (Bergquist et al., 2010). One well-studied form of homeostatic plasticity at the fly NMJ is called presynaptic homeostatic potentiation (PHP). Here, pharmacologic or genetic perturbations to postsynaptic glutamate receptors trigger a retrograde signaling system that leads to a compensatory enhancement in presynaptic neurotransmitter release that maintains muscle excitation (Frank, 2014). We applied the glutamate receptor antagonist PhTx to WT and $p k c 53 E$ mutant NMJs. In both cases, mEPSP amplitudes were reduced by $\sim 50 \%$ due to $\mathrm{PhTx}$ blocking a subset of the postsynaptic glutamate receptors, as expected (Fig. 4G,H). A homeostatic increase in presynaptic glutamate release (quantal content) was observed in both WT and pkc53E that restored synaptic strength (EPSP amplitude) to baseline levels (Fig. 4G,H), demonstrating that PHP was robustly expressed. We also assessed PHP expression after PhTx application in the eight remaining synaptic growth mutants as well and found that in all cases PHP was robustly expressed (Table 4-1, available at https://doi.org/ 10.1523/JNEUROSCI.2601-18.2019.t4-1). Thus, the ability (or inability) to express adaptive plasticity in the context of synaptic growth does not occlude the competence to transduce
PHP signaling, consistent with independent homeostatic mechanisms that can be superimposed and balanced (Goel et al., 2019b).

Enhanced postsynaptic receptor abundance compensates for reduced presynaptic release in WRNexo mutants

We next focused on the undergrowth mutant WRNexo. WRNexo encodes Werner's exonuclease, so named because mutations in the human homolog cause Werner's syndrome, a disease resulting in premature aging due to DNA damage (Muftuoglu et al., 2008). Null mutations in WRNexo have been generated and characterized in the context of DNA repair in Drosophila (Bolterstein et al., 2014). However, roles for WRNexo in synaptic growth or function have not been reported, nor have they been characterized at the NMJ. WRNexo mutants exhibit significant reductions in synaptic growth, with bouton numbers reduced by $\sim 50 \%$ compared to WT controls, with a concomitant reduction in active zone numbers labeled by BRP (Fig. $5 A, B$ ). However, EPSP amplitude in WRNexo mutants was similar to WT (Fig. $5 C, D)$. Quantification of mEPSP amplitude revealed a significant increase in WRNexo mutants compared with WT, resulting in a corresponding reduction in quantal content (Fig. $5 C, D)$. Together, this suggests that while presynaptic neurotransmitter release is reduced in accordance to reduced synaptic growth in WRNexo mutants, an increase in the postsynaptic responsiveness to neurotransmitter was sufficient to maintain normal synaptic strength. 
A

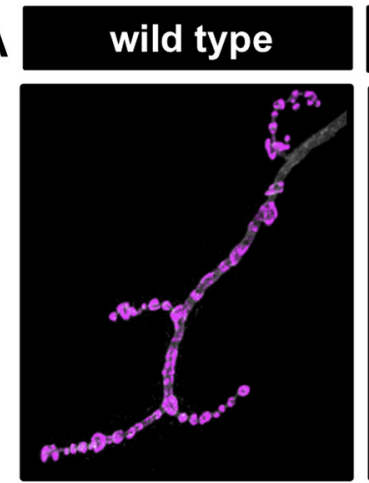

C

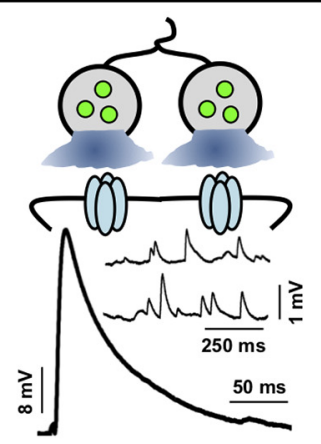

E
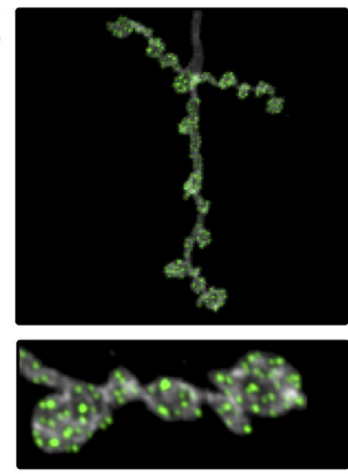
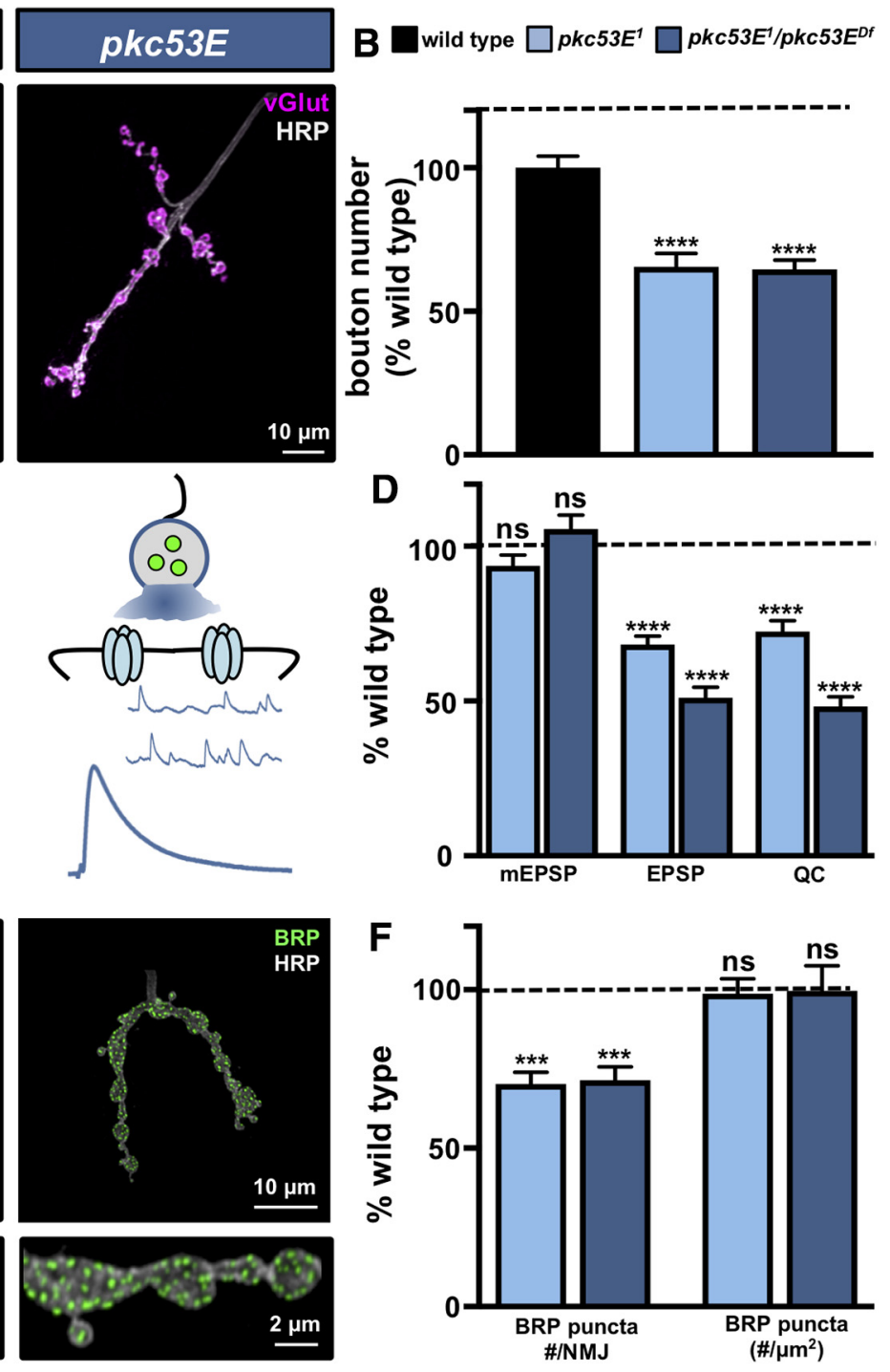
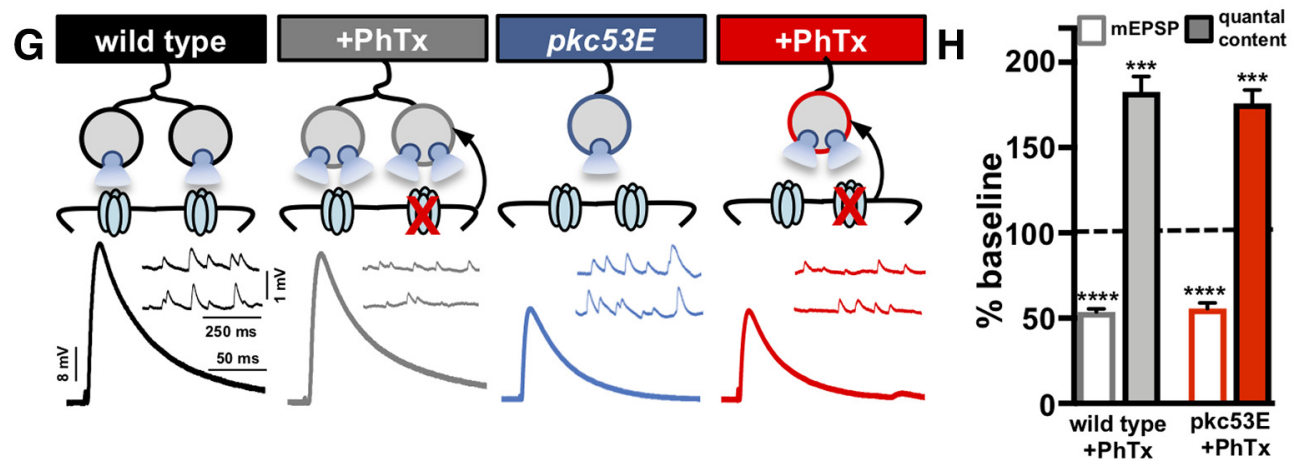

Figure 4. Presynaptic neurotransmitter release scales with reduced bouton and active zone number in $p k c 53 E$ mutants. $A$, Representative muscle 4 NMJ images of WT ( $w^{1718}$ ) and $p k c 53 E$ mutants in trans with a deficiency ( $\left.p k c 53 E^{1} / p k c 53 E^{D f(2 R) P 803}\right)$ immunostained with anti-HRP and anti-vGlut. B, Quantification of bouton number in the indicated genotypes normalized to WT values. $C$, Schematic and representative electrophysiological traces of mEPSPs and EPSPs in the indicated genotypes illustrating reduced synaptic strength and no evidence for compensatory adaptions to presynaptic neurotransmitter release or postsynaptic sensitivity to neurotransmitter. Green dots in the schematic represent BRP puncta reduced in parallel with bouton numbers in $p k c 53 E$. $\mathbf{D}$, Quantification of $\mathrm{mEPSP}$, EPSP, and quantal content values in pkc53E mutants normalized as a percentage of WT. $\boldsymbol{E}$, Representative images of NMJs immunostained with anti-HRP and the anti-BRP (presynaptic active zone marker), with individual boutons shown at higher magnification (insets below). $\boldsymbol{F}$, Quantification of total BRP puncta number per NMJ shows a concomitant reduction with bouton number and no significant change in BRP puncta density. G, Schematic and representative traces of recordings from WT and pkc53E mutants before and following PhTx application. Diminished mEPSP amplitudes are observed following PhTx application, whereas EPSP amplitudes are maintained at baseline levels due to a homeostatic increase in presynaptic neurotransmitter release (quantal content). $\boldsymbol{H}$, Quantification of mEPSP and quantal content values following PhTx application normalized as a percentage of baseline values (no PhTx treatment) in the indicated genotypes. Error bars indicate \pm SEM. One-way ANOVA test was performed, followed by a Tukey's multiple-comparison test: ${ }^{* * *} p \leq 0.001 ;{ }^{* * * *} p \leq 0.0001$. ns, Not significant $(p>0.05)$. Detailed statistical information (mean values, SEM, $n, p$ ) is shown in Table 4-1 (available at https://doi.org/10.1523/JNEUROSCI.2601-18.2019.t4-1). 

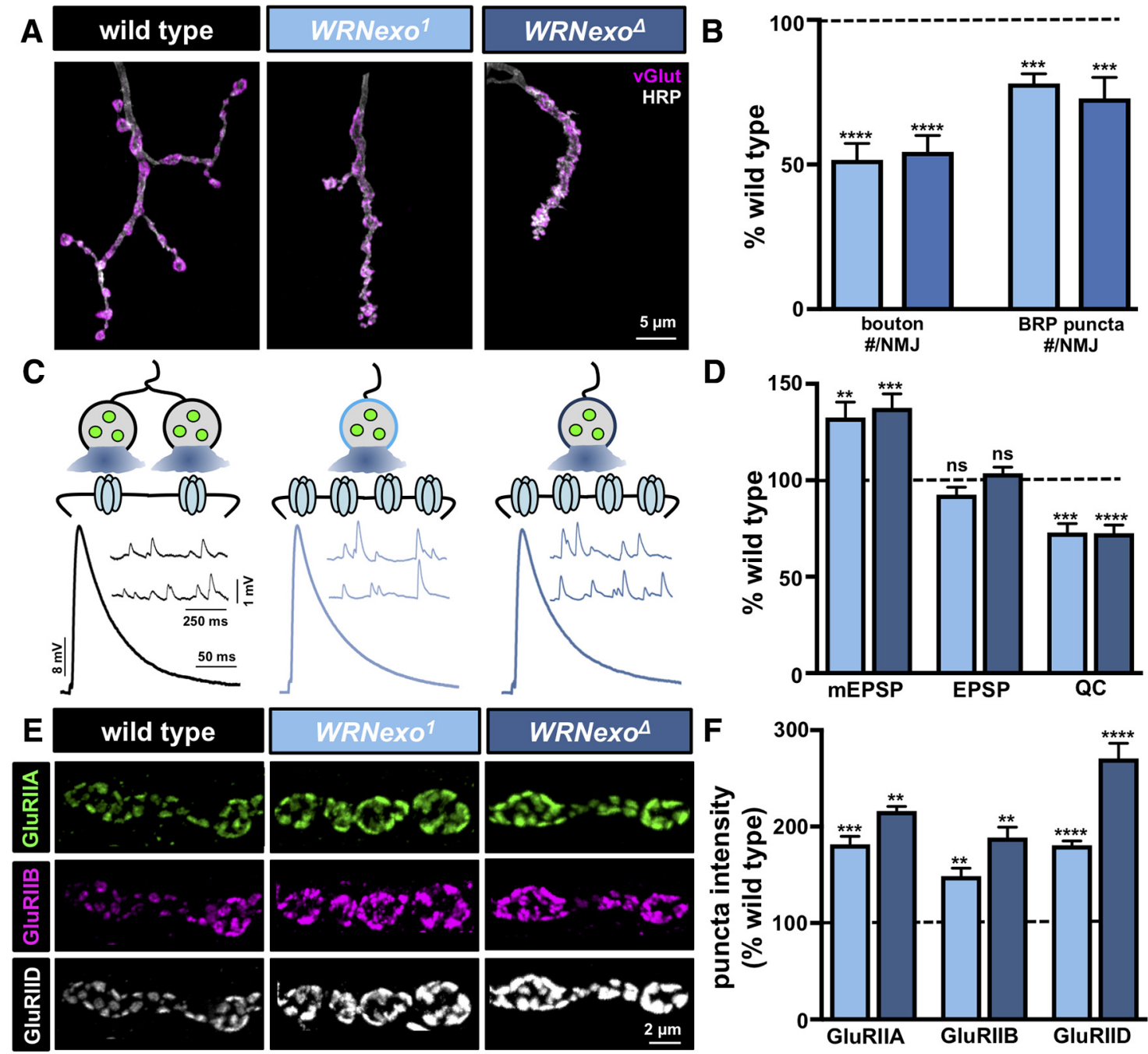

Figure 5. Increased postsynaptic receptor levels compensate for reduced presynaptic neurotransmitter release in WRNexo mutants. $A$, Representative images of muscle 4 NMJs in WT, WRNexo mutants $\left(\right.$ WRNexO $\left.{ }^{M / 13095}\right)$, and WRNexo null mutants $\left(\right.$ WRNexO $\left.{ }^{\mathrm{D}}\right)$, immunostained with anti-HRP and anti-vGlut. B, Quantification of bouton numbers and BRP puncta numbers in WRNexo mutants normalized as a percentage of WT. C, Representative mEPSP and EPSP traces in the indicated genotypes. The schematic illustrates that enhanced levels of postsynaptic glutamate receptor levels offset reduced presynaptic release in WRNexo mutants with reduced bouton and BRP puncta numbers. D, Quantification of mEPSP, EPSP, and quantal content values in the indicated genotypes normalized as a percentage of WT. $\boldsymbol{E}$, Representative images of NMJs immunostained with antibodies against three postsynaptic glutamate receptor subunits (GluRIIA; GluRIIB; GluRIID). $\boldsymbol{F}$, Quantification of sum puncta fluorescence intensity of each receptor subunit reveals enhanced levels of both GluRIIA- and GluRIIB-containing receptors in WRNexo. Error bars indicate \pm SEM. One-way ANOVA test was performed, followed by a Tukey's multiple-comparison test: ${ }^{* *} p \leq 0.01 ;{ }^{* * *} p \leq 0.001 ;{ }^{* * * *} p \leq 0.0001$. ns, Not significant $(p>0.05)$. Detailed statistical information (mean values, SEM, $n, p$ ) is shown in Table 4-1 (available at https://doi.org/10.1523/JNEUROSCI.2601-18.2019.t4-1).

At the Drosophila NMJ, two glutamate receptor subtypes, GluRIIA-containing and GluRIIB-containing, mediate the response to synaptically released glutamate (DiAntonio, 2006). Three essential glutamate receptors (GluRIIC, GluRIID, and GluRIIE) are core components of both receptor complexes and incorporate either GluRIIA or GluRIIB subunits (Qin et al., 2005; DiAntonio, 2006). The majority of neurotransmission is driven by GluRIIA-containing receptors due to their slower desensitization kinetics and larger current amplitudes (Petersen et al., 1997; DiAntonio et al., 1999; Han et al., 2015). Given the increase in mEPSP amplitude observed in WRNexo mutants, we examined the state of glutamate receptors in more detail. We costained NMJs with antibodies against GluRIIA, GluRIIB, and GluRIID and assessed the synaptic localization of these receptor subunits while also quantifying immunofluorescence levels (Fig. $5 E, F$ ). Although we did not observe any major differences in the localization of receptors at the NMJ, we did find a significant increase in GluRIIA, GluRIIB, and GluRIID subunit levels in WRNexo mutants (Fig. $5 E, F$ ). This suggests that the enhanced abundance of postsynaptic glutamate receptors served to increase sensitivity to neurotransmitter in WRNexo mutants. In turn, this compensated for reduced synaptic growth and glutamate release to maintain proper muscle excitation.

\section{Increased bouton area maintains stable synapse number in} cont and $G \gamma 30 a$ mutants

Next, we characterized the two remaining synaptic undergrowth mutants, contactin (cont), a cell adhesion molecule involved in septate junction organization between glia and neurons (FaivreSarrailh et al., 2004), and G $\gamma 30 A$, a heterotrimeric G-protein subunit (Schillo et al., 2004). Interestingly, despite an $\sim 60 \%$ reduction in bouton number compared with WT (Fig. $6 A, B$ ), these two mutants did not exhibit any apparent changes in synaptic physiology (Fig. 6C,D). mEPSP amplitudes were similar to WT in both mutants, which implies that a presynaptic change in either active zone number and/or release probability likely compen- 


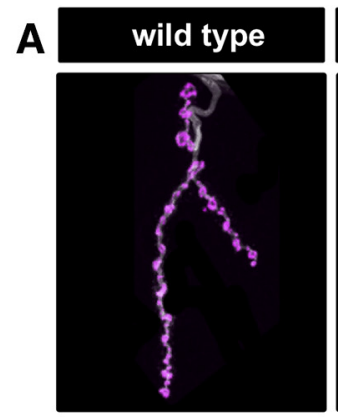

C
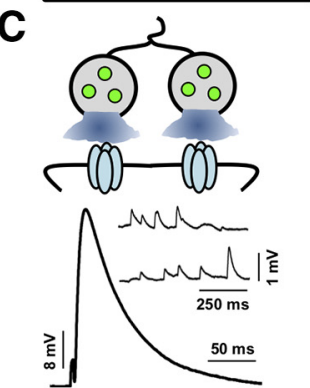

E

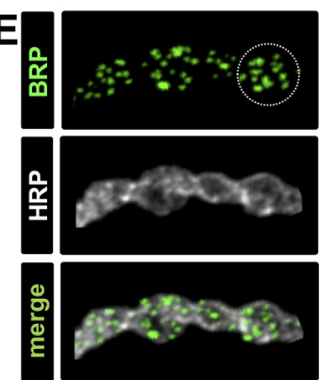

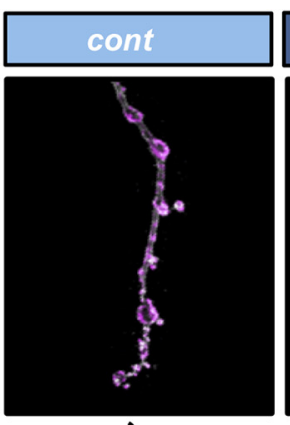
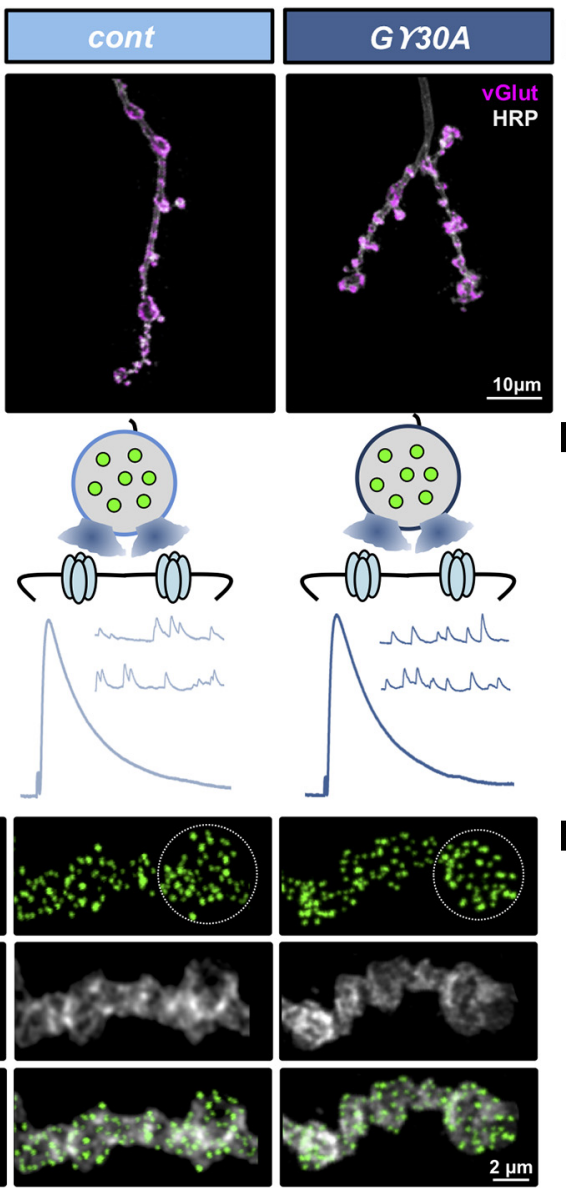
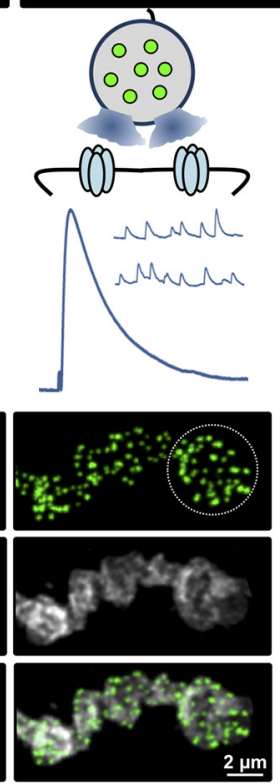

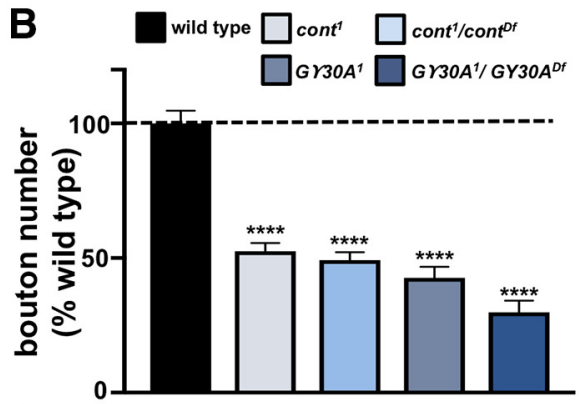

D
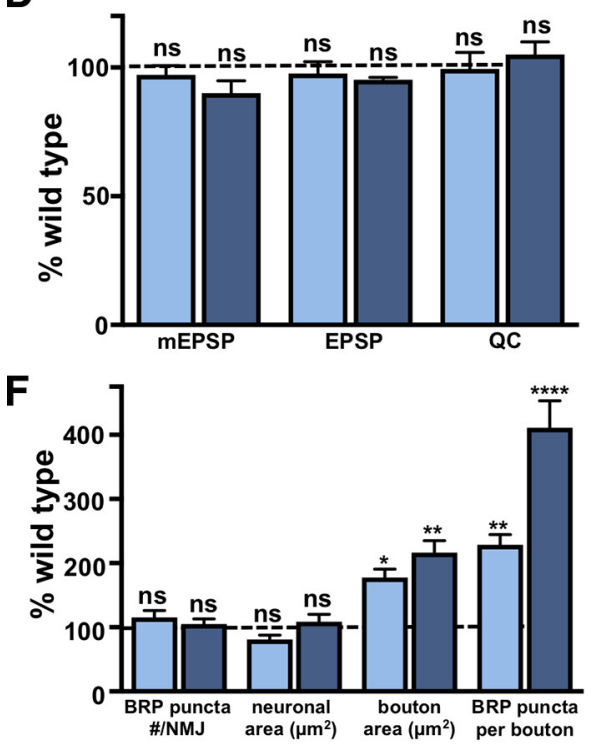

Figure 6. Increased bouton size compensates for reduced bouton number in cont and $G \gamma 30 A$ mutants. $A$, Representative images of muscle 4 NMJs in WT, cont, and $G \gamma 30 A$ mutants in trans with deficiencies (cont: cont ${ }^{1} /$ cont $^{D f(3 R) B S C 146} ; G \gamma 30 A: G \gamma 30 A^{1} / G \gamma 30 A{ }^{D f(2 L) E D 680}$ ) immunostained with anti-HRP and anti-vGlut. $\boldsymbol{B}$, Bouton numbers per NMJ in the indicated genotypes normalized as a percentage of WT values. $C$, Representative mEPSP and EPSP traces in the indicated genotypes. The schematic illustrates an enhancement in bouton area resulting in more release sites per bouton in cont and $G \gamma 30 \mathrm{~A}$ mutants. D, Quantification of mEPSP, EPSP, and quantal content values in the indicated genotypes normalized as a percentage of WT values. $\boldsymbol{E}$, Representative images of individual boutons from the indicated genotypes immunostained with anti-BRP and anti-HRP. White circle outlines a single bouton. The increased area of individual boutons and number of BRP puncta within each bouton is apparent in cont and $G \gamma 30 \mathrm{~A}$ mutants. $\boldsymbol{F}$, Quantification of the indicated synaptic parameters in the indicated genotypes normalized as a percentage of WT values. Total neuronal membrane area is unchanged in cont and $G \gamma 30 \mathrm{~A}$ mutants due to an increase in the average area of individual boutons. Hence, a significant increase in the number of BRP puncta per bouton is observed. Error bars indicate \pm SEM. ANOVA test was performed, followed by a Tukey's multiple-comparison test: ${ }^{*} p \leq 0.05 ;{ }^{* *} p \leq 0.01{ }^{* * * *} p \leq 0.0001$. ns, Not significant $(p>0.05)$. Detailed statistical information (mean values, SEM, $\left.n, p\right)$ is shown in Table 4-1 (available at https://doi.org/10.1523/JNEUROSCI.2601-18.2019.t4-1).

sated for reduced bouton number to maintain stable levels of presynaptic neurotransmitter release.

We therefore quantified the number of BRP puncta per NMJ in cont and G $330 \mathrm{~A}$ mutants. Surprisingly, immunostaining of $\mathrm{BRP}$ revealed that total puncta number per NMJ were similar in both cont and G $\gamma 30 A$ mutants compared with WT (Fig. 6E,F). Further analysis found that while bouton numbers were indeed reduced, individual boutons were significantly enlarged in area in these mutants (Fig. 6E,F). Thus, although cont and $G \gamma 30 A$ were defined as synaptic undergrowth mutants based on our bouton counting assay, increased bouton area conserved total neuronal membrane surface area (Fig. $6 F$ ). Consistently, quantification of BRP puncta per bouton revealed a significant increase in both cont and Gr30A (Fig. 6E,F), demonstrating that active zone number scaled with the enhanced NMJ membrane and area of individual boutons. Thus, despite a reduction in overall bouton number, increased synapse number per bouton was sufficient to maintain total synapse number per NMJ, and global neurotransmitter output, in both cont and G $330 \mathrm{~A}$ undergrowth mutants.
Reduced active zone area in synaptic overgrowth mutants compensates for increased active zone numbers

Finally, we characterized synaptic function and structure in the five remaining synaptic overgrowth mutants. This category harbored mutations in diverse genes encoding the G-protein coupled receptor mangetout $(m t t)$; the WD repeat domain protein 62 ( $w d r 62)$; the kainate receptor ekar; the calcium-activated protein phosphatase calcineurin B2 (canB2); and the endoplasmic reticulum stress gene receptor expression enhancing protein (reep). Despite the diverse functions of these genes (Table 1-2, available at https://doi.org/10.1523/JNEUROSCI.2601-18.2019.t1-2), they shared a common $40 \%-50 \%$ increase in the number of synaptic boutons per NMJ but stable synaptic strength (Fig. 7A-C). Electrophysiological analysis revealed no significant changes in mEPSP amplitude, EPSP amplitude, or quantal content (Fig. 7 B,E; Table 4-1, available at https://doi.org/10.1523/JNEUROSCI.2601-18. 2019.t4-1). This suggests that the postsynaptic sensitivity to neurotransmitter was not impacted in these mutants and implies a change in synapse number and/or release probability likely com- 

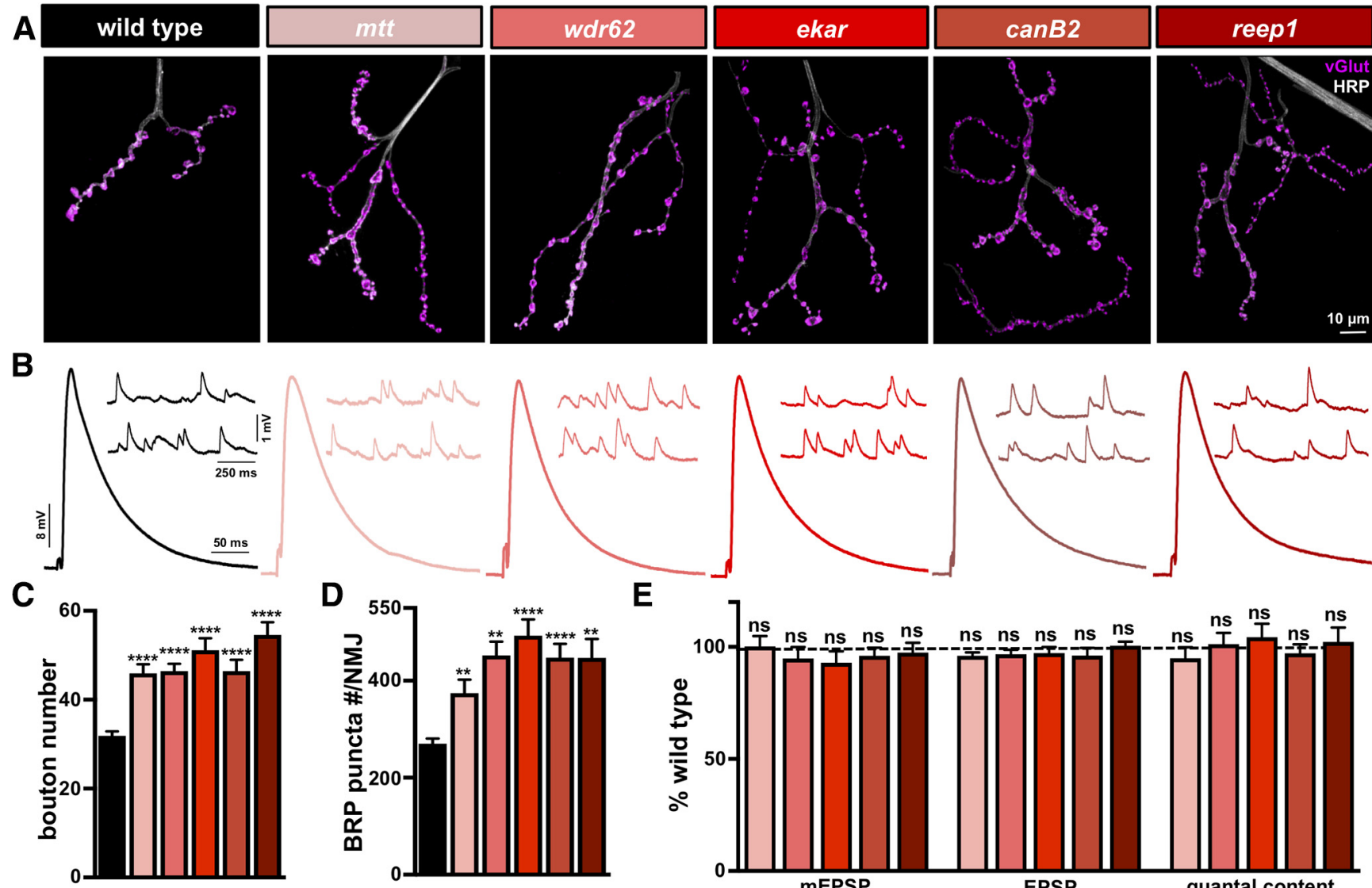

E

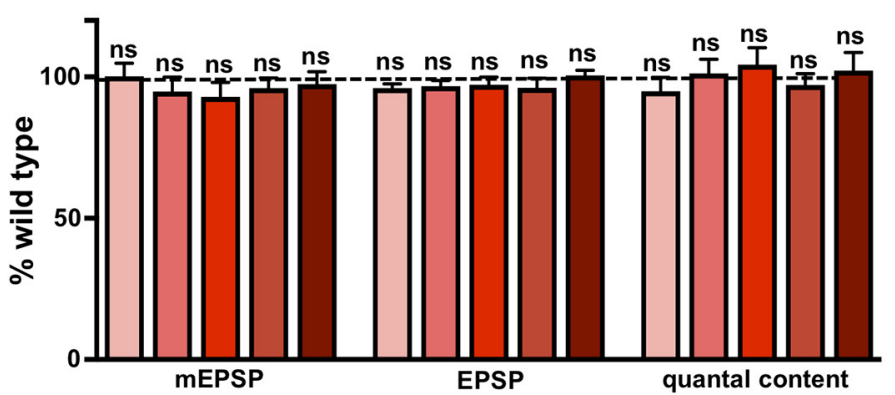

Figure 7. Mutants with enhanced synaptic growth exhibit a concomitant increase in active zone number yet stable levels of synaptic strength. $A$, Representative images of muscle 4 NMJs in WT,

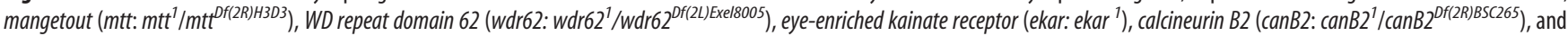
receptor expression enhancing protein (reep1: reep ${ }^{1} /$ reep $1^{\text {Df(2R)WI345 }}$ ) mutants immunostained with anti-HRP and anti-vGlut. $\boldsymbol{B}$, Representative EPSP and mEPSP traces showing no significant changes in the overgrowth mutants compared with WT. Quantification of bouton numbers ( $\boldsymbol{C}$ and BRP puncta number per NMJ (D) in the indicated genotypes reveals a significant increase in both parameters compared with WT. E, Quantification of mEPSP, EPSP, and quantal content values in the indicated genotypes normalized as a percentage of WT. Despite enhanced bouton and active zone number per NMJ in the overgrowth mutants, no significant change in presynaptic neurotransmitter release (quantal content) is observed. Error bars indicate \pm SEM. One-way ANOVA test was performed, followed by a Tukey's multiple-comparison test: ${ }^{* *} p \leq 0.01 ;{ }^{* * * *} p \leq 0.0001$. ns, Not significant $(p>0.05)$. Detailed statistical information (mean values, SEM, $\left.n, p\right)$ is shown in Table 4-1 (available at https://doi.org/10.1523/JNEUROSCI.2601-18.2019.t4-1).

pensated for the increased bouton number shared in these mutants.

Next, we quantified the total number of BRP puncta per NMJ in these overgrowth mutants. We found an increase in total BRP puncta number per NMJ that correlated with the enhanced synaptic growth observed in each overgrowth mutant (Fig. 7C,D). Correspondingly, we observed no major differences in bouton size, leading to a parallel increase in total neuronal membrane surface area per NMJ and no change in BRP puncta density (Table 4-1, available at https://doi.org/10.1523/JNEUROSCI.260118.2019.t4-1). Hence, BRP puncta number essentially scaled with bouton number in the overgrowth mutants, in contrast to the undergrowth mutants detailed in Figure 6. This suggests that a reduction in release probability per active zone likely stabilized synaptic strength in these mutants.

The size and abundance of material at individual active zones can vary, and several studies have found that these properties correlate with release probability (Murthy et al., 2001; Matz et al., 2010; Holderith et al., 2012). At the Drosophila NMJ, there is considerable heterogeneity in the size and intensity of the active zone scaffold BRP and other active zone components (Guerrero et al., 2005; Peled and Isacoff, 2011; Ehmann et al., 2014; Akbergenova et al., 2018; Gratz et al., 2019). Furthermore, recent studies have shown that increasing intensity and size of active zone components, including BRP, Unc13, and the $\mathrm{Ca}^{2+}$ channel Cac, predict increased release probability during baseline transmission and after homeostatic plasticity (Weyhersmüller et al., 2011; Goel et al., 2017, 2019b; Li et al., 2018a; Böhme et al., 2019; Gratz et al., 2019). We therefore considered that while the total number of BRP puncta per NMJ was increased in the overgrowth mutants, there might have been a corresponding change in the area and/or intensity of each puncta that contributed to their modulation of release probability.

Analysis of individual BRP puncta revealed a significant reduction in the mean area of BRP puncta in all five synaptic overgrowth mutants (Fig. 8A,B; Table 4-1, available at https:// doi.org/10.1523/JNEUROSCI.2601-18.2019.t4-1). Indeed, the average BRP puncta area scaled with total BRP puncta number per NMJ in WT and in the synaptic overgrowth mutants (Fig. 8C; $\left.R^{2}=0.27, p=0.0006\right)$. However, the total abundance of BRP per NMJ, reflected in the sum fluorescence intensity of BRP puncta across an entire NMJ, was not significantly different between WT and the five overgrowth mutants (Fig. 8D; Table 4-1, available at https://doi.org/10.1523/JNEUROSCI.2601-18.2019.t4-1). In contrast, such a scaling of BRP puncta intensity with BRP number was not observed in the undergrowth mutants $p k c 53 E$ G $\gamma 30 \mathrm{~A}$, or cont (Table 4-1, available at https://doi.org/10.1523/JNEUROSCI. 2601-18.2019.t4-1). Interestingly, a similar scaling of BRP size 

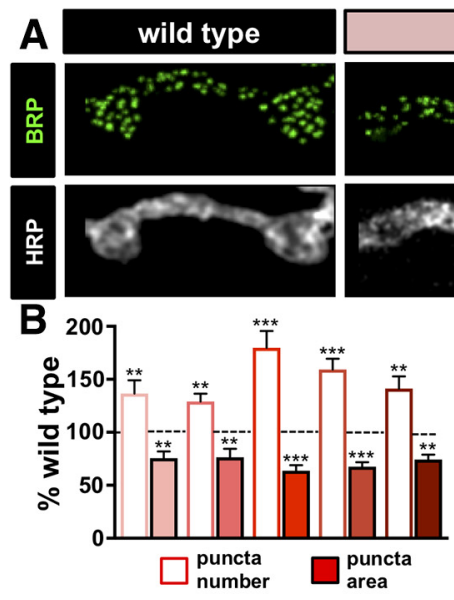
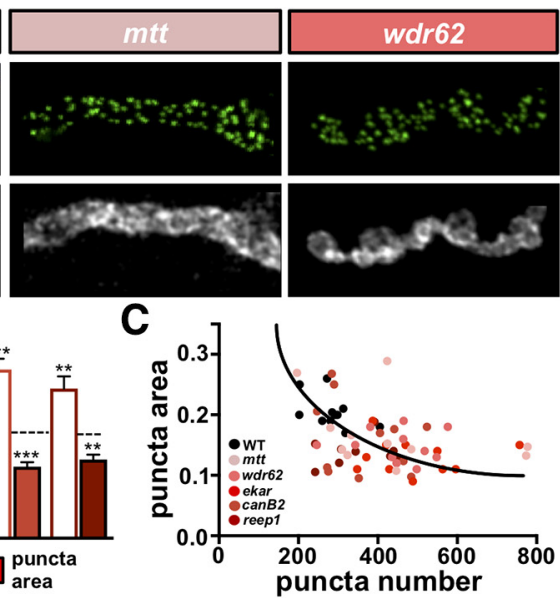
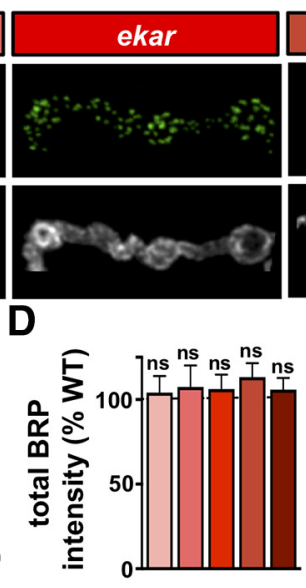

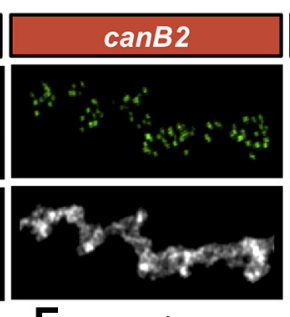

E
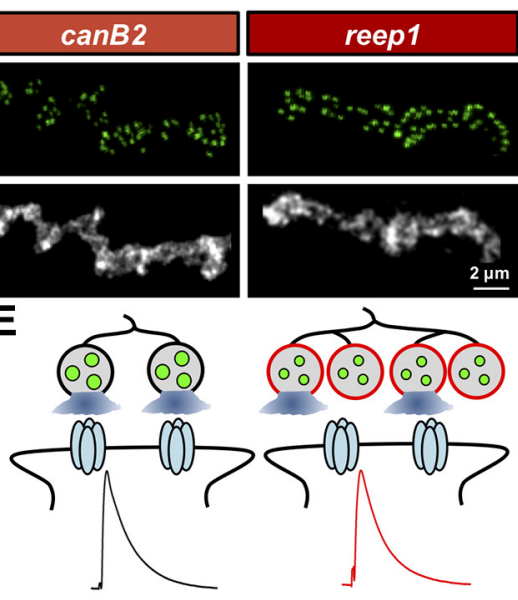

Figure 8. Active zone area is reduced in mutants with enhanced synaptic growth. $A$, Representative images of individual boutons from WT and the overgrowth mutants immunostained with anti-BRP and anti-HRP. B, Quantification of BRP puncta number and BRP puncta area in the indicated genotypes normalized to WT values. While both bouton and BRP puncta numbers are increased in the overgrowth mutants, a reduction in the average area of each BRP puncta is observed. $C$, Average BRP puncta area plotted as a function of average BRP puncta number per NMJ in the indicated genotypes demonstrates a homeostatic scaling of average BRP puncta area with total BRP number per NMJ, represented by the curve fitted to the data points $\left(R^{2}=0.27\right)$. ${ }^{* *} p=0.0006 . D_{\text {, }}$ Quantification of total BRP puncta fluorescence intensity per NMJ in the indicated genotypes, suggesting that the total abundance of BRP per NMJ remains unchanged in the overgrowth mutants compared with WT. $\boldsymbol{E}$, Schematic illustrating increased bouton and BRP puncta numbers in the overgrowth mutants with a reduction in the area of individual BRP puncta. This results in reduced release probability per active zone and per bouton to stabilize NMJ strength. Error bars indicate \pm SEM. One-way ANOVA test was performed, followed by a Tukey's multiple-comparison test: ${ }^{* *} p \leq 0.01 ;{ }^{* * *} p \leq 0.001$. ns, Not significant $(p>0.05$ ). Detailed statistical information (mean values, SEM, $n, p$ ) is shown in Table 4-1 (available at https://doi.org/10.1523/JNEUROSCI.2601-18.2019.t4-1).

and number while conserving total BRP intensity per NMJ was demonstrated in the dramatic synaptic overgrowth mutant endophilin (Goel et al., 2019b), suggesting that this process may be a more general compensatory adaptation to synaptic exuberance. Thus, an apparent tuning of active zone size may have compensated for increased number to reduce release probability per active zone and maintain synaptic strength in the overgrowth mutants identified from the screen.

\section{Discussion}

Through a forward genetic screen of $\sim 300$ mutants, we have identified genes required for proper regulation of synaptic growth and neurotransmission. This approach has revealed several new mutations and RNAi lines that exhibit aberrant synaptic growth and function, and further analysis demonstrated that the strength of neurotransmission was regulated independently of synaptic growth. Together, these data imply the existence of a homeostat that stabilizes global synaptic strength while permitting substantial flexibility in synaptic growth. Our analysis has defined three adaptive mechanisms that operate to maintain synaptic strength when synaptic growth is dramatically altered.

Integration of structural and functional plasticity at synapses A complex repertoire of genes and processes work together to tune and coordinate synaptic growth, structure, and function during development. The strength of our large-scale screening approach lies in identifying mutants with major deviations in synaptic growth and using these as informative tools to reveal developmental solutions used by the NMJ to homeostatically maintain synaptic strength. We have implied that the primary perturbation in the mutants characterized is a defect in synaptic growth, and the homeostatic response involves changes in receptor abundance, presynaptic morphology, or active zone structure. Although it is formally possible that in each of the mutants studied, the deviation in synaptic growth may be the homeostatic response to changes in presynaptic or postsynaptic structure, we favor the former interpretation for the following reasons. First, none of the genes linked to the synaptic growth mutants share obvious functions in receptor trafficking, neuronal morphogenesis, or active zone development (Table 1-2, available at https:// doi.org/10.1523/JNEUROSCI.2601-18.2019.t1-2), yet all utilize similar adaptions to morphology or active zone structure. Second, baseline levels of NMJ strength persist when synaptic growth is enhanced by manipulations that extend the third instar stage in an otherwise WT larvae (Miller et al., 2012). Third, hyperinnervation or hypoinnervation to the fly NMJ triggers both presynaptic and postsynaptic forms of homeostatic plasticity (Davis and Goodman, 1998a; Goel and Dickman, 2018), which parallel the mechanisms of active zone remodeling and postsynaptic receptor enhancement that we describe here. Finally, stable synaptic strength persists in a variety of other mutations that impact synaptic growth (Verstreken et al., 2002; Marie et al., 2004; Dickman et al., 2006; Goel et al., 2019b), while no change in synaptic growth has been described in NMJ models of homeostatic potentiation or depression (Frank et al., 2006; Dickman and Davis, 2009; Gavino et al., 2015; Wang et al., 2016, 2018; Li et al., 2018a). Thus, while it is established that synaptic function can be homeostatically tuned in response to both excess neurotransmitter release and diminished postsynaptic receptors (Li et al., 2018a), developmental changes in synaptic growth may be an additional perturbation capable of inducing similar homeostatic responses.

There is emerging evidence that both homeostatic and Hebbian forms of plasticity share common genes and signaling networks (Vitureira and Goda, 2013; Keck et al., 2017; Turrigiano, 2017; Li et al., 2019). While the Drosophila NMJ is built for stability and has proven to be a powerful model to investigate glutamatergic transmission and homeostatic plasticity, contrasting forms of Hebbian plasticity are less obvious at this synapse. Hence, mutations of genes with specialized functions in nonglutamatergic synaptic transmission or Hebbian plasticity are unlikely to reveal major phenotypes using the screening strategy we used. However, a variety of genes were identified with more subtle roles in regulating synaptic growth and baseline function 
(Table 1-1, available at https://doi.org/10.1523/JNEUROSCI. 2601-18.2019.t1-1). Mutations in one gene, pkc53E, exhibited reduced synaptic growth and a parallel reduction in transmission, consistent with a scaling model of synaptic growth and transmission. However, our characterization of the remaining synaptic growth mutants revealed evidence for homeostatic adaptations that stabilized synaptic strength across variations in NMJ growth. In the case of the undergrowth mutants cont and $G \gamma 30 \mathrm{~A}$, increased size of individual boutons led to a conservation of both neuronal membrane and active zone number to maintain synaptic strength. Interestingly, there is evidence that the size of individual boutons is inversely correlated with total numbers at the Drosophila NMJ (Pennetta et al., 2002; Miech et al., 2008; Pawson et al., 2008; Mosca et al., 2012; Goel et al., 2019b). Therefore, adjusting the morphology and size of boutons is one adaptive strategy that may generally serve to enable flexibility in synaptic growth while maintaining stable total synapse numbers.

\section{Homeostatic scaling of glutamate receptor abundance and active zone size}

We identified a homeostatic scaling of postsynaptic glutamate receptor abundance that offset reduced presynaptic neurotransmitter release in one synaptic undergrowth mutant. Specifically, WRNexo mutants exhibited reduced synaptic growth with a concomitant reduction in presynaptic active zone number and neurotransmitter output. However, this diminished presynaptic efficacy was offset by a compensatory increase in postsynaptic receptors. This phenomenon parallels homeostatic scaling of postsynaptic glutamate receptors following manipulations to activity in mammalian central neurons (Turrigiano et al., 1998; Aoto et al., 2008; Turrigiano, 2008; Chen et al., 2014). While glutamate receptors are dynamically regulated in central neurons during both Hebbian and homeostatic forms of plasticity (PérezOtaño and Ehlers, 2005; Herring and Nicoll, 2016; Chowdhury and Hell, 2018), receptors at the NMJ are much less dynamic. Glutamate receptors have half-lives of $\sim 24 \mathrm{~h}$ at the Drosophila NMJ (Rasse et al., 2005), similar to the relatively slow turnover of cholinergic receptors at the mammalian NMJ (Salpeter and Harris, 1983). However, there is intriguing evidence that the normally stable postsynaptic receptors at the NMJ can be dynamically regulated in response to presynaptic activity (Schmid et al., 2008; Ljaschenko et al., 2013), injury and disease (Rich and Lichtman, 1989; Palma et al., 2011; Xiong and Collins, 2012; Perry et al., 2017; Goel and Dickman, 2018), and even altered innervation (Davis and Goodman, 1998a; Goel and Dickman, 2018). Similar phenomena occur following injury in the mammalian CNS (Dunn, 2015). Thus, NMJs may be endowed with an underappreciated degree of latent receptor plasticity that can be revealed in response to homeostatic challenges, including synaptic undergrowth.

We identified an apparent homeostatic scaling of active zone size in all five synaptic overgrowth mutants. In contrast to the undergrowth mutants, no changes in bouton size or the postsynaptic sensitivity to neurotransmitter were observed, and active zone number scaled with enhanced synaptic growth. In principle, a variety of compensatory changes in postsynaptic receptors, presynaptic morphology, and/or synapse number could have been homeostatically regulated to maintain synaptic strength. However, all five mutants shared an apparent reduction in the size and intensity of the active zone scaffold BRP, indicative of a functional reduction in release probability of individual active zones. Interestingly, the abundance of active zone proteins can predict release probability due to enhancement in calcium influx and the size of the readily releasable synaptic vesicle pool (Weyhersmüller et al., 2011; Matkovic et al., 2013; Colloway et al., 2015; Held et al., 2016; Gratz et al., 2019). Indeed, a positive correlation between the size and intensity of active zone components and release probability has been demonstrated at the Drosophila NMJ (Guerrero et al., 2005; Peled and Isacoff, 2011; Akbergenova et al., 2018) as well as at mammalian central synapses (Murthy et al., 2001; Matz et al., 2010; Holderith et al., 2012; Glebov et al., 2017). Furthermore, BRP can be remodeled during presynaptic homeostatic potentiation to enhance release probability at active zones (Weyhersmüller et al., 2011; Goel et al., 2017, 2019b; Böhme et al., 2019; Gratz et al., 2019). Therefore, the reduction in active zone size observed in the overgrowth mutants likely reduces release probability at individual release sites to maintain global neurotransmitter output at the NMJ, an adaptation most dramatically illustrated in endophilin mutants (Goel et al., 2019b). In addition, presynaptic homeostatic potentiation can be induced and expressed locally to enable compartmentalized changes in release probability at a subset of NMJ synapses (Li et al., 2018b). More generally, remodeling of active zone structure is an attractive mechanism that might homeostatically tune both local and global presynaptic efficacy to stabilize neurotransmission while still permitting flexibility during synaptic growth and pruning.

In the CNS, a variety of mechanisms homeostatically scale axonal and dendritic structure and arborization to compensate for altered activity. For example, a homeostatic remodeling of dendritic arborization in the fly visual system is observed in response to chronically elevated or reduced activity (Yuan et al., 2011), and adaptive structural alterations at synapses have been observed during sleep/wake behavior (Bushey et al., 2011; Diering et al., 2017; de Vivo et al., 2017). Similarly, compensatory changes in the structure and number of dendritic spines are observed in response to imbalances in excitation and inhibition in the CNS (Butz et al., 2009; Holtmaat and Svoboda, 2009; Fu and Zuo, 2011; Bosch and Hayashi, 2012; Wong and Guo, 2013). Parallel adaptations to the axon initial segment and release probability at presynaptic terminals counteract homeostatic challenges (Murthy et al., 2001; Kuba et al., 2010; Vitureira and Goda, 2013). Our findings on the interplay between synaptic growth and function underscore the diverse mechanisms that homeostatically stabilize global synaptic strength while permitting dynamic flexibility in the growth of synapses.

\section{References}

Akbergenova Y, Cunningham KL, Zhang YV, Weiss S, Littleton JT (2018) Characterization of developmental and molecular factors underlying release heterogeneity at Drosophila synapses. eLife 7:e38268.

Aoto J, Nam CI, Poon MM, Ting P, Chen L (2008) Synaptic signaling by all-trans retinoic acid in homeostatic synaptic plasticity. Neuron 60:308320 .

Atwood HL, Govind CK, Wu CF (1993) Differential ultrastructure of synaptic terminals on ventral longitudinal abdominal muscles in Drosophila larvae. J Neurobiol 24:1008-1024.

Bao H, Berlanga ML, Xue M, Hapip SM, Daniels RW, Mendenhall JM, Alcantara AA, Zhang B (2007) The atypical cadherin flamingo regulates synaptogenesis and helps prevent axonal and synaptic degeneration in Drosophila. Mol Cell Neurosci 34:662-678.

Baram TZ (2012) The brain, seizures and epilepsy throughout life: understanding a moving target. Epilepsy Curr 12:7-12.

Bergquist S, Dickman DK, Davis GW (2010) A hierarchy of cell intrinsic and target-derived homeostatic signaling. Neuron 66:220-234.

Böhme MA, McCarthy AW, Grasskamp AT, Beuschel CB, Goel P, Jusyte M, Laber D, Huang S, Rey U, Petzoldt AG, Lehmann M, Göttfert F, Haghighi P, Hell SW, Owald D, Dickman D, Sigrist SJ, Walter AM (2019) Rapid 
active zone remodeling consolidates presynaptic potentiation. Nat Commun 10:1085.

Bolterstein E, Rivero R, Marquez M, McVey M (2014) The Drosophila Werner exonuclease participates in an exonuclease-independent response to replication stress. Genetics 197:643-652.

Bosch M, Hayashi Y (2012) Structural plasticity of dendritic spines. Curr Opin Neurobiol 22:383-388.

Brusich DJ, Spring AM, Frank CA (2015) A single-cross, RNA interferencebased genetic tool for examining the long-term maintenance of homeostatic plasticity. Front Cell Neurosci 9:107.

Bushey D, Tononi G, Cirelli C (2011) Sleep and synaptic homeostasis: structural evidence in Drosophila. Science 332:1576-1581.

Butz M, Wörgotter F, van Ooyen A (2009) Activity-dependent structural plasticity. Brain Res Rev 60:287-305.

Chen L, Lau AG, Sarti F (2014) Synaptic retinoic acid signaling and homeostatic synaptic plasticity. Neuropharmacology 78:3-12.

Chen X, Ma W, Zhang S, Paluch J, Guo W, Dickman DK (2017) The BLOC-1 subunit pallidin facilitates activity-dependent synaptic vesicle recycling. eNeuro 4:1-18.

Chowdhury D, Hell JW (2018) Homeostatic synaptic scaling: molecular regulators of synaptic AMPA-type glutamate receptors. F1000Res 7:234.

Cohen-Cory S (2002) The developing synapse: construction and modulation of synaptic structures and circuits. Science 298:770-776.

Colloway N, Gouzer G, Xue M, Ryan TA (2015) The active-zone protein Munc13 controls the use dependence of presynaptic voltage-gated calcium channels. eLife 4:e07728.

Daniels RW, Collins CA, Gelfand MV, Dant J, Brooks ES, Krantz DE, DiAntonio A (2004) Increased expression of the Drosophila vesicular glutamate transporter leads to excess glutamate release and a compensatory decrease in quantal content. J Neurosci 24:10466-10474.

Darnell JC, Van Driesche SJ, Zhang C, Hung KY, Mele A, Fraser CE, Stone EF, Chen C, Fak JJ, Chi SW, Licatalosi DD, Richter JD, Darnell RB (2011) FMRP stalls ribosomal translocation on mRNAs linked to synaptic function and autism. Cell 146:247-261.

Davis GW, Goodman CS (1998a) Synapse-specific control of synaptic efficacy at the terminals of a single neuron. Nature 392:82-86.

Davis GW, Goodman CS (1998b) Genetic analysis of synaptic development and plasticity: homeostatic regulation of synaptic efficacy. Curr Opin Neurobiol 8:149-156.

Del Castillo JL, Katz B (1954) Quantal components of the end-plate potential. J Physiol 124:560-573.

de Vivo L, Bellesi M, Marshall W, Bushong EA, Ellisman MH, Tononi G, Cirelli C (2017) Ultrastructural evidence for synaptic scaling across the wake/sleep cycle. Science 355:507-510.

DiAntonio A (2006) Glutamate receptors at the Drosophila neuromuscular junction. Intl Rev Neurobiol 75:165-179.

DiAntonio A, Petersen SA, Heckmann M, Goodman CS (1999) Glutamate receptor expression regulates quantal size and quantal content at the Drosophila neuromuscular junction. J Neurosci 19:3023-3032.

Dickman DK, Davis GW (2009) The schizophrenia susceptibility gene dysbindin controls synaptic homeostasis. Science 326:1127-1130.

Dickman DK, Horne JA, Meinertzhagen IA, Schwarz TL (2005) A slowed classical pathway rather than kiss-and-run mediates endocytosis at synapses lacking synaptojanin and endophilin. Cell 123:521-533.

Dickman DK, Lu Z, Meinertzhagen IA, Schwarz TL (2006) Altered synaptic development and active zone spacing in endocytosis mutants. Curr Biol 16:591-598.

Diering GH, Nirujogi RS, Roth RH, Worley PF, Pandey A, Huganir RL (2017) Homerla drives homeostatic scaling-down of excitatory synapses during sleep. Science 355:511-515.

Dunn FA (2015) Photoreceptor ablation initiates the immediate loss of glutamate receptors in postsynaptic bipolar cells in retina. J Neurosci 35: 2423-2431.

Ehmann N, van de Linde S, Alon A, Ljaschenko D, Keung XZ, Holm T, Rings A, DiAntonio A, Hallermann S, Ashery U, Heckmann M, Sauer M, Kittel RJ (2014) Quantitative super-resolution imaging of Bruchpilot distinguishes active zone states. Nat Commun 5:4650.

Faivre-Sarrailh C, Banerjee S, Li J, Hortsch M, Laval M, Bhat MA (2004) Drosophila contactin, a homolog of vertebrate contactin, is required for septate junction organization and paracellular barrier function. Development 131:4931-4942.
Forrest MP, Parnell E, Penzes P (2018) Dendritic structural plasticity and neuropsychiatric disease. Nat Rev Neurosci 19:215-234.

Frank CA (2014) Homeostatic plasticity at the Drosophila neuromuscular junction. Neuropharmacology 78:63-74.

Frank CA, Kennedy MJ, Goold CP, Marek KW, Davis GW (2006) Mechanisms underlying the rapid induction and sustained expression of synaptic homeostasis. Neuron 52:663-677.

Fu M, Zuo Y (2011) Experience-dependent structural plasticity in the cortex. Trends Neurosci 34:177-187.

Gavino MA, Ford KJ, Archila S, Davis GW (2015) Homeostatic synaptic depression is achieved through a regulated decrease in presynaptic calcium channel abundance. eLife 4:e05473.

Gilman SR, Iossifov I, Levy D, Ronemus M, Wigler M, Vitkup D (2011) Rare de novo variants associated with autism implicate a large functional network of genes involved in formation and function of synapses. Neuron 70:898-907.

Glebov OO, Jackson RE, Winterflood CM, Owen DM, Barker EA, Doherty P, Ewers H, Burrone J (2017) Nanoscale structural plasticity of the active zone matrix modulates presynaptic function. Cell Rep 18:2715-2728.

Goel P, Dickman D (2018) Distinct homeostatic modulations stabilize reduced postsynaptic receptivity in response to presynaptic DLK signaling. Nat Commun 9:1856.

Goel P, Li X, Dickman D (2017) Disparate postsynaptic induction mechanisms ultimately converge to drive the retrograde enhancement of presynaptic efficacy. Cell Rep 21:2339-2347.

Goel P, Li X, Dickman D (2019a) Estimation of the readily releasable synaptic vesicle pool at the Drosophila larval neuromuscular junction. Bio Protoc 9:e3127.

Goel P, Bergeron DD, Böhme M, Nunnelly L, Lehmann M, Buser C, Walter AM, Sigrist SJ, Dickman DK (2019b) Homeostatic scaling of active zone scaffolds maintains global synaptic strength. J Cell Biol DOI: 10.1083/ jcb.201807165.

Gratz SJ, Goel P, Bruckner JJ, Hernandez RX, Khateeb K, Macleod G, Dickman D, O'Connor-Giles KM (2019) Endogenous tagging reveals differential regulation of $\mathrm{Ca}^{2+}$ channels at single AZs during presynaptic homeostatic potentiation and depression. J Neurosci 39:3068-3118.

Guerrero G, Reiff DF, Agarwal G, Ball RW, Borst A, Goodman CS, Isacoff EY (2005) Heterogeneity in synaptic transmission along a Drosophila larval motor axon. Nat Neurosci 8:1188-1196.

Han TH, Dharkar P, Mayer ML, Serpe M (2015) Functional reconstitution of Drosophila melanogaster NMJ glutamate receptors. Proc Natl Acad Sci U S A 112:6182-6187.

Held RG, Liu C, Kaeser PS (2016) ELKS controls the pool of readily releasable vesicles at excitatory synapses through its $\mathrm{N}$-terminal coiled-coil domains. eLife 5:e14862.

Herring BE, Nicoll RA (2016) Long-term potentiation: from CaMKII to AMPA receptor trafficking. Annu Rev Physiol 78:351-365.

Holderith N, Lorincz A, Katona G, Rózsa B, Kulik A, Watanabe M, Nusser Z (2012) Release probability of hippocampal glutamatergic terminals scales with the size of the active zone. Nat Neurosci 15:988-997.

Holmes GL, Ben-Ari Y (1998) Seizures in the developing brain. Neuron 21:1231-1234.

Holtmaat A, Svoboda K (2009) Experience-dependent structural synaptic plasticity in the mammalian brain. Nat Rev Neurosci 10:647-658.

Jurado S, Goswami D, Zhang Y, Molina AJ, Südhof TC, Malenka RC (2013) LTP requires a unique postsynaptic SNARE fusion machinery. Neuron 77:542-558

Keck T, Toyoizumi T, Chen L, Doiron B, Feldman DE, Fox K, Gerstner W, Haydon PG, Hübener M, Lee HK, Lisman JE, Rose T, Sengpiel F, Stellwagen D, Stryker MP, Turrigiano GG, van Rossum MC (2017) Integrating Hebbian and homeostatic plasticity: the current state of the field and future research directions. Philos Trans R Soc Lond B Biol Sci 372: 20160158

Kikuma K, Li X, Kim D, Sutter D, Dickman DK (2017) Extended synaptotagmin localizes to presynaptic ER and promotes neurotransmission and synaptic growth in Drosophila. Genetics 207:993-1006.

Kiragasi B, Wondolowski J, Li Y, Dickman DK (2017) A presynaptic glutamate receptor subunit confers robustness to neurotransmission and homeostatic potentiation. Cell Rep 19:2694-2706.

Kittel RJ, Wichmann C, Rasse TM, Fouquet W, Schmidt M, Schmid A, Wagh DA, Pawlu C, Kellner RR, Willig KI, Hell SW, Buchner E, Heckmann M, 
Sigrist SJ (2006) Bruchpilot promotes active zone assembly, $\mathrm{Ca}^{2+}$ channel clustering, and vesicle release. Science 312:1051-1054.

Kuba H, Oichi Y, Ohmori H (2010) Presynaptic activity regulates $\mathrm{Na}(+)$ channel distribution at the axon initial segment. Nature 465:1075-1078.

Li J, Park E, Zhong LR, Chen L (2019) Homeostatic synaptic plasticity as a metaplasticity mechanism: a molecular and cellular perspective. Curr Opin Neurobiol 54:44-53.

Li X, Goel P, Wondolowski J, Paluch J, Dickman D (2018a) A glutamate homeostat controls the presynaptic inhibition of neurotransmitter release. Cell Rep 23:1716-1727.

Li X, Goel P, Chen C, Angajala V, Chen X, Dickman DK (2018b) Synapsespecific and compartmentalized expression of presynaptic homeostatic potentiation. eLife 7:e34338.

Liebl FL, Werner KM, Sheng Q, Karr JE, McCabe BD, Featherstone DE (2006) Genome-wide P-element screen for Drosophila synaptogenesis mutants. J Neurobiol 66:332-347.

Lisman JE, Raghavachari S, Tsien RW (2007) The sequence of events that underlie quantal transmission at central glutamatergic synapses. Nat Rev Neurosci 8:597-609.

Ljaschenko D, Ehmann N, Kittel RJ (2013) Hebbian plasticity guides maturation of glutamate receptor fields in vivo. Cell Rep 3:1407-1413.

Lnenicka GA, Mellon D Jr (1983a) Changes in electrical properties and quantal current during growth of identified muscle fibres in the crayfish. J Physiol 345:261-284.

Lnenicka GA, Mellon D Jr (1983b) Transmitter release during normal and altered growth of identified muscle fibres in the crayfish. J Physiol 345: 285-296.

Marie B, Sweeney ST, Poskanzer KE, Roos J, Kelly RB, Davis GW (2004) Dap160/Intersectin scaffolds the periactive zone to achieve high-fidelity endocytosis and normal synaptic growth. Neuron 43:207-219.

Matkovic T, Siebert M, Knoche E, Depner H, Mertel S, Owald D, Schmidt M, Thomas U, Sickmann A, Kamin D, Hell SW, Bürger J, Hollmann C, Mielke T, Wichmann C, Sigrist SJ (2013) The Bruchpilot cytomatrix determines the size of the readily releasable pool of synaptic vesicles. J Cell Biol 202:667-683.

Matz J, Gilyan A, Kolar A, McCarvill T, Krueger SR (2010) Rapid structural alterations of the active zone lead to sustained changes in neurotransmitter release. Proc Natl Acad Sci U S A 107:8836-8841.

Miech C, Pauer HU, He X, Schwarz TL (2008) Presynaptic local signaling by a canonical wingless pathway regulates development of the Drosophila neuromuscular junction. J Neurosci 28:10875-10884.

Miller DL, Ballard SL, Ganetzky B (2012) Analysis of synaptic growth and function in Drosophila with an extended larval stage. J Neurosci 32: $13776-13786$.

Mosca TJ, Hong W, Dani VS, Favaloro V, Luo L (2012) Trans-synaptic teneurin signalling in neuromuscular synapse organization and target choice. Nature 484:237-241.

Muftuoglu M, Oshima J, von Kobbe C, Cheng WH, Leistritz DF, Bohr VA (2008) The clinical characteristics of Werner syndrome: molecular and biochemical diagnosis. Hum Genet 124:369-377.

Müller M, Pym EC, Tong A, Davis GW (2011) Rab3-GAP controls the progression of synaptic homeostasis at a late stage of vesicle release. Neuron 69:749-762.

Murthy VN, Schikorski T, Stevens CF, Zhu Y (2001) Inactivity produces increases in neurotransmitter release and synapse size. Neuron 32:673682.

Natarajan R, Trivedi-Vyas D, Wairkar YP (2013) Tuberous sclerosis complex regulates Drosophila neuromuscular junction growth via the TORC2/Akt pathway. Hum Mol Genet 22:2010-2023.

Newman ZL, Hoagland A, Aghi K, Worden K, Levy SL, Son JH, Lee LP, Isacoff EY (2017) Input-specific plasticity and homeostasis at the Drosophila larval neuromuscular junction. Neuron 93:1388-1404.e10.

Palma E, Inghilleri M, Conti L, Deflorio C, Frasca V, Manteca A, Pichiorri F, Roseti C, Torchia G, Limatola C, Grassi F, Miledi R (2011) Physiological characterization of human muscle acetylcholine receptors from ALS patients. Proc Natl Acad Sci U S A 108:20184-20188.

Pawson C, Eaton BA, Davis GW (2008) Formin-dependent synaptic growth; evidence that Dlar signals via diaphanous to modulate synaptic actin and dynamic pioneer microtubules. J Neurosci 28:11111-11123.

Peled ES, Isacoff EY (2011) Optical quantal analysis of synaptic transmission in WT and rab3-mutant Drosophila motor axons. Nat Neurosci 14: 519-526.
Pennetta G, Hiesinger PR, Fabian-Fine R, Meinertzhagen IA, Bellen HJ (2002) Drosophila VAP-33A directs bouton formation at neuromuscular junctions in a dosage-dependent manner. Neuron 35:291-306.

Pérez-Otaño I, Ehlers MD (2005) Homeostatic plasticity and NMDA receptor trafficking. Trends Neurosci 28:229-238.

Perry S, Han Y, Das A, Dickman D (2017) Homeostatic plasticity can be induced and expressed to restore synaptic strength at neuromuscular junctions undergoing ALS-related degeneration. Hum Mol Genet 26:4153-4167.

Petersen SA, Fetter RD, Noordermeer JN, Goodman CS, DiAntonio A (1997) Genetic analysis of glutamate receptors in Drosophila reveals a retrograde signal regulating presynaptic transmitter release. Neuron 19:1237-1248.

Pielage J, Fetter RD, Davis GW (2005) Presynaptic spectrin is essential for synapse stabilization. Curr Biol 15:918-928.

Pozo K, Goda Y (2010) Unraveling mechanisms of homeostatic synaptic plasticity. Neuron 66:337-351.

Qin G, Schwarz T, Kittel RJ, Schmid A, Rasse TM, Kappei D, Ponimaskin E, Heckmann M, Sigrist SJ (2005) Four different subunits are essential for expressing the synaptic glutamate receptor at neuromuscular junctions of Drosophila. J Neurosci 25:3209-3218.

Rasse TM, Fouquet W, Schmid A, Kittel RJ, Mertel S, Sigrist CB, Schmidt M, Guzman A, Merino C, Qin G, Quentin C, Madeo FF, Heckmann M, Sigrist SJ (2005) Glutamate receptor dynamics organizing synapse formation in vivo. Nat Neurosci 8:898-905

Reddy-Alla S, Böhme MA, Reynolds E, Beis C, Grasskamp AT, Mampell MM, Maglione M, Jusyte M, Rey U, Babikir H, McCarthy AW, Quentin C, Matkovic T, Bergeron DD, Mushtaq Z, Göttfert F, Owald D, Mielke T, Hell SW, Sigrist SJ, et al. (2017) Stable positioning of Unc13 restricts synaptic vesicle fusion to defined release sites to promote synchronous neurotransmission. Neuron 95:1350-1364.e12.

Riccomagno MM, Kolodkin AL (2015) Sculpting neural circuits by axon and dendrite pruning. Annu Rev Cell Dev Biol 31:779-805.

Rich MM, Lichtman JW (1989) In vivo visualization of pre- and postsynaptic changes during synapse elimination in reinnervated mouse muscle. J Neurosci 5:1781-1805.

Salpeter MM, Harris R (1983) Distribution and turnover rate of acetylcholine receptors throughout the junction folds at a vertebrate neuromuscular junction. J Cell Biol 96:1781-1785.

Sando R 3rd, Gounko N, Pieraut S, Liao L, Yates J 3rd, Maximov A (2012) HDAC4 governs a transcriptional program essential for synaptic plasticity and memory. Cell 151:821-834.

Schillo S, Belusic G, Hartmann K, Franz C, Kühl B, Brenner-Weiss G, Paulsen R, Huber A (2004) Targeted mutagenesis of the farnesylation site of Drosophila Ggammae disrupts membrane association of the $\mathrm{G}$ protein betagamma complex and affects the light sensitivity of the visual system. J Biol Chem 279:36309-36316.

Schmid A, Hallermann S, Kittel RJ, Khorramshahi O, Frölich AM, Quentin C, Rasse TM, Mertel S, Heckmann M, Sigrist SJ (2008) Activity-dependent site-specific changes of glutamate receptor composition in vivo. Nat Neurosci 11:659-666.

Schuster CM, Davis GW, Fetter RD, Goodman CS (1996) Genetic dissection of structural and functional components of synaptic plasticity: II. Fasciclin II controls presynaptic structural plasticity. Neuron 17:655667.

Serretti A, Fabbri C (2013) Shared genetics among major psychiatric disorders. Lancet 381:1339-1341.

Sigrist SJ, Reiff DF, Thiel PR,Steinert JR, Schuster CM (2003) Experiencedependent strengthening of Drosophila neuromuscular junctions. J Neurosci 23:6546-6556.

Stewart BA, Atwood HL, Renmger JJ, Wang J, Wu CF (1994) Improved stability of Drosophila larval neuromuscular preparations in haemolymphlike physiological solutions. J Comp Physiol 175:179-191.

Thomson SR, Seo SS, Barnes SA, Louros SR, Muscas M, Dando O, Kirby C, Wyllie DJ, Hardingham GE, Kind PC, Osterweil EK (2017) Cell-typespecific translation profiling reveals a novel strategy for treating fragile $\mathrm{X}$ syndrome. Neuron 95:550-563.e5.

Turrigiano GG (2008) The self-tuning neuron: synaptic scaling of excitatory synapses. Cell 135:422-435.

Turrigiano GG (2017) The dialectic of Hebb and homeostasis. Philos Trans R Soc Lond B Biol Sci 372:20160258. 
Turrigiano GG, Leslie KR, Desai NS, Rutherford LC, Nelson SB (1998) Activity-dependent scaling of quantal amplitude in neocortical neurons. Nature 391:892-896.

Verstreken P, Kjaerulff O, Lloyd TE, Atkinson R, Zhou Y, Meinertzhagen IA, Bellen HJ (2002) Endophilin mutations block clathrin-mediated endocytosis but not neurotransmitter release. Cell 109:101-112.

Vitureira N, Goda Y (2013) The interplay between hebbian and homeostatic synaptic plasticity. J Cell Biol 203:175-186.

Wagh DA, Rasse TM, Asan E, Hofbauer A, Schwenkert I, Dürrbeck H, Buchner S, Dabauvalle MC, Schmidt M, Qin G, Wichmann C, Kittel R, Sigrist SJ, Buchner E (2006) Bruchpilot, a protein with homology to ELKS/ CAST, is required for structural integrity and function of synaptic active zones in Drosophila. Neuron 49:833-844.

Wang X, Pinter MJ, Rich MM (2016) Reversible recruitment of a homeostatic reserve pool of synaptic vesicles underlies rapid homeostatic plasticity of quantal content. J Neurosci 36:828-836.

Wang X, McIntosh JM, Rich MM (2018) Muscle nicotinic acetylcholine receptors may mediate trans-synaptic signaling at the mouse neuromuscular junction. J Neurosci 38:1725-1736.

Weyhersmüller A, Hallermann S, Wagner N, Eilers J (2011) Rapid active zone remodeling during synaptic plasticity. J Neurosci 31:6041-6052.

Wondolowski J, Dickman D (2013) Emerging links between homeostatic synaptic plasticity and neurological disease. Front Cell Neurosci 7:223.

Wong M, Guo D (2013) Dendritic spine pathology in epilepsy: cause or consequence? Neuroscience 251:141-150.

Xiong X, Collins CA (2012) A conditioning lesion protects axons from degeneration via the Wallenda/DLK MAP kinase signaling cascade. J Neurosci 32:610-615.

Yuan Q, Xiang Y, Yan Z, Han C, Jan LY, Jan YN (2011) Light-induced structural and functional plasticity in Drosophila larval visual system. Science 333:1458-1462.

Zoghbi HY, Bear MF (2012) Synaptic dysfunction in neurodevelopmental disorders associated with autism and intellectual disabilities. Cold Spring Harbor Perspect Biol 4:a009886. 